\title{
Regularity and geometric estimates for minima of discontinuous functionals
}

\author{
Raimundo Leitão and Eduardo V. Teixeira
}

\begin{abstract}
In this paper we study nonnegative minimizers of general degenerate elliptic functionals, $\int F(X, u, \nabla u) d X \rightarrow$ min, for variational kernels $F$ that are discontinuous in $u$ with discontinuity of order $\sim \chi_{\{u>0\}}$. The Euler-Lagrange equation is therefore governed by a nonhomogeneous, degenerate elliptic equation with free boundary between the positive and the zero phases of the minimizer. We show optimal gradient estimate as well as nondegeneracy of minima. We also address weak and strong regularity properties of the free boundary. We show the set $\{u>0\}$ has locally finite perimeter and that the reduced free boundary, $\partial_{\text {red }}\{u>0\}$, has $\mathcal{H}^{n-1}$-total measure. For more specific problems that arise in jet flows, we show the reduced free boundary is locally the graph of a $C^{1, \gamma}$ function.
\end{abstract}

\section{Introduction}

Given a bounded smooth domain $\Omega \subset \mathbb{R}^{n}$ and a nonnegative function $\phi \in W^{1, p}(\Omega)$ $\cap L^{\infty}(\bar{\Omega}), 2 \leq p<n$, we study regularity and fine geometric properties of solutions to the minimization problem

$$
\min \left\{\int_{\Omega} F(X, u, \nabla u) d X: u \in W_{\phi}^{1, p}(\Omega)\right\},
$$

where $W_{\phi}^{1, p}(\Omega)$ denotes the Sobolev space of all functions in $L^{p}$ with distributional derivatives in $L^{p}$ and trace value $\phi$. The variational kernel $F: \Omega \times \mathbb{R} \times \mathbb{R}^{n} \rightarrow \mathbb{R}$, will be written as $F(X, u, \xi)=G(X, \xi)+g(X, u)$ and it will satisfy the following structural conditions:

(G1) For all $\xi \in \mathbb{R}^{n}$, the mapping $X \mapsto G(X, \xi)$ is continuous.

(G2) There exists a positive constant $0<\lambda$ such that

$$
\lambda|\xi|^{p} \leq G(X, \xi) \leq \lambda^{-1}|\xi|^{p}
$$

Mathematics Subject Classification (2010): Primary 35R35, 35B65; Secondary 35J70.

Keywords: Discontinuous functionals, free boundary problems, degenerate elliptic equations. 
(G3) For almost all $X \in \Omega$, the mapping $\xi \mapsto G(X, \xi)$ is strictly convex, differentiable and satisfies

$$
G(X, t \xi)=|t|^{p} G(X, \xi), \quad t \in \mathbb{R}, \xi \in \mathbb{R}^{n} .
$$

(G4) There exist constants $0<\delta<1$ and $C_{\mathcal{A}}>0$ such that

$$
X \mapsto \mathcal{A}(X, \xi) \in C^{\delta}\left(\Omega^{\prime}\right), \quad \sup _{\xi \in \mathbb{R}^{n}}\|\mathcal{A}(X, \xi)\|_{C^{\delta}} \leq C_{\mathcal{A}}
$$

for all subdomain $\Omega^{\prime} \subset \Omega$, where $\mathcal{A}(X, \xi):=\nabla_{\xi} G(X, \xi)$.

(g1) The function $g$ is defined by

$$
g(X, u)=f(X)\left(u^{+}\right)^{m}+Q \chi_{\{u>0\}}, \quad 1 \leq m<p,
$$

where $f$ is measurable, $-K \leq f \leq K$, for some $K>0$ and $Q$ is $C^{0, \beta}$-continuous, $0<\kappa<Q<\kappa^{-1}$ for some $\kappa>0$.

A prototypical kernel to keep in mind is

$$
F(X, u, \xi)=|\xi|^{p-2} A(X) \xi \cdot \xi+f(X)\left(u^{+}\right)^{m}+Q \chi_{\{u>0\}},
$$

for a positive definite matrix $A$ with continuous coefficients. Motivations come from the study of jet flow and cavity problems, among many other applications. For notational convenience, we label the functional appearing in the minimization problem $(1.1)$ by $\mathfrak{F}: W_{\varphi}^{1, p}(\Omega) \rightarrow \mathbb{R}$, i.e., hereafter

$$
\mathfrak{F}(u):=\int_{\Omega} F(X, u, \nabla u) d X .
$$

Also, any positive constant $C=C\left(n, p, m, \lambda, \phi, \kappa, K, \delta, C_{\mathcal{A}}, \Omega\right)$ that depends only on dimension and the parameter constants of the problem will henceforth be called a universal constant. We call a solution of the homogeneous problem

$$
\operatorname{div}(\mathcal{A}(X, \nabla h))=0 \quad \text { in } B_{r}\left(X_{0}\right) \subset \Omega .
$$

an $\mathcal{A}$-harmonic functions. The key feature of the functional $\mathfrak{F}$ is that it is discontinuous with respect to $u$, thus the well established classical theory of the calculus of variations is not suitable for treating such problems. In fact, at a minimum $u$, the functional $\mathfrak{F}$ presents discontinuity for small perturbations near points on the, in principle unknown, set $\partial\{u>0\}$. Such a discontinuity reflects a lack of smoothness of $u$ across the boundary of its zero level surface.

The study of the variational problem (1.1) goes back to the fundamental work of Alt and Caffarelli, [1], which provides a thorough analysis of this problem for $p=2$, $f \equiv 0$, and $A(X)=$ Id in (1.2). Danielli and Petrosyan in [6] developed the corresponding Alt and Caffarelli theory for the $p$-Laplacian, i.e., $f \equiv 0$ and $A(X)=\mathrm{Id}$.

In this paper we study the variational problem (1.1) in full generality, providing existence, regularity, and geometric properties of certain heterogeneous free boundary problems governed by degenerate elliptic equations. The results from this work are new even for the Poisson type equation $m=1$. They also yield new results even in the linear setting $p=2$. 
In Section 3 we show there is a minimum for the functional $\mathfrak{F}$ and that this minimum is nonnegative and continuous in $\Omega$. We further show that within the set of positivity, $u$ satisfies the desired Euler-Lagrange equation

$$
\operatorname{div}\left(\nabla_{\xi} G(X, \nabla u)\right)=m f(X) u^{m-1}, \quad \text { in }\{u>0\},
$$

in the sense of distributions. Under natural structural assumptions on the kernel $G$, $u$ is $C^{1, \alpha}$ in its set of positivity. Nevertheless, due to the discontinuity of $\mathfrak{F}$ near free boundary points, $\nabla u$ jumps from positive values to zero through $\partial\{u>0\}$. Therefore, the optimal regularity possible for a minimum is Lipschitz continuity. Such a result is established in Section 4. By Lipschitz regularity, we conclude that $u$ grows linearly away from the free boundary. However, from energy considerations, we actually show that $u$ grows precisely linearly on $\partial\{u>0\}$. This is important geometric information that provides access to finer geometric-measure features of the free boundary. In fact, in Section 5 we show that

$$
\Lambda:=\operatorname{div}\left(\nabla_{\xi} G(X, \nabla u)\right)-m f(X) u^{m-1},
$$

defines a nonnegative measure supported along the free boundary. We further show that the set of positivity of $u,\{u>0\}$, is locally a set of finite perimeter. A finer property is actually shown: we verify that

$$
\mathcal{H}^{n-1}\left(\partial\{u>0\} \cap B_{r}(Z)\right) \sim r^{n-1},
$$

for any ball $B_{r}(Z)$ centered at a free boundary point. In particular we conclude the reduced free boundary, $\partial_{\text {red }}\{u>0\}$ has total $\mathcal{H}^{n-1}$-Hausdorff measure.

In the last Section we address smoothness of the (reduced) free boundary for the heterogeneous, quasilinear cavity problem

$$
\left\{\begin{aligned}
\operatorname{div}(A(X) \nabla u) & =m f(X) u^{m-1}, & & \text { in }\{u>0\}, \\
\langle A \nabla u, \nabla u\rangle & =Q, & & \text { on } \partial\{u>0\} \cap \Omega,
\end{aligned}\right.
$$

where the matrix $A$ is Lipschitz and positive definite. We show the free boundary is a $C^{1, \gamma}$ smooth hypersurface, up to a possible $\mathcal{H}^{n-1}$ negligible set, providing therefore a classical solution to the corresponding quasilinear Bernoulli type problem.

Acknowledgement. This paper is part of the first author's doctoral thesis at the Department of Mathematics at Universidade Federal do Ceará, Brazil. Both authors would like to express their gratitude to this institution for such a pleasant and productive scientific atmosphere.

\section{Preliminaries}

In this section we gather some preliminary results that we will repeatedly throughout the article. We begin with the classical criterion of Ladyzhenskaya and Ural'tseva to estimate the $L^{\infty}$-norm of a function $u \in W_{\phi}^{1, p}(\Omega)$ with boundary values $\phi \in L^{\infty}(\partial \Omega)$ for $1<p \leq n$. 
Lemma 2.1. Let $u \in W^{1, p}(\Omega), 1<p \leq n$, be such that $\|u\|_{L^{\infty}(\partial \Omega)}<\infty$. For $j \geq j_{0} \geq\|u\|_{L^{\infty}(\partial \Omega)}$, let $A_{j}:=\{u>j\}$. If $u$ satisfies

$$
\int_{A_{j}}|\nabla u|^{p} \leq \gamma j^{\alpha} \mathcal{L}^{n}\left(A_{j}\right)^{1-p / n+\varepsilon}
$$

where $\gamma>0, \varepsilon>0$ and $0 \leq \alpha \leq \varepsilon+p$, then there is a constant $C>0$ depending only on $\gamma, \alpha, p, n, \varepsilon, j_{0}$, and $\|u\|_{L^{1}\left(A_{j_{0}}\right)}$ such that

$$
\|u\|_{L^{\infty}(\Omega)} \leq C .
$$

See [13], Chapter 2, Page 71, Lemma 5.2, for a proof of Lemma 2.1. Clearly, the case $p>n$ is a direct consequence of the Sobolev inequality.

The following is a basic fact: if $h$ is a solution in $W_{v}^{1, p}\left(B_{r}\left(X_{0}\right)\right)$ of equation (1.3), then $h$ is the unique minimizer of the functional

$$
\mathfrak{F}(w):=\int_{B_{r}\left(X_{0}\right)}\langle\mathcal{A}(X, \nabla w), \nabla w\rangle d X,
$$

among functions $w \in W_{0}^{1, p}\left(B_{1}\right)+v$. Thus, we have

$$
\begin{aligned}
\lambda \int_{B_{r}\left(X_{0}\right)}|\nabla h|^{p} d X & \leq \int_{B_{r}\left(X_{0}\right)}\langle\mathcal{A}(X, \nabla h), \nabla h\rangle d X \\
& \leq \int_{B_{r}\left(X_{0}\right)}\langle\mathcal{A}(X, \nabla v), \nabla v\rangle d X \leq \lambda^{-1} \int_{B_{r}\left(X_{0}\right)}|\nabla v|^{p} d X .
\end{aligned}
$$

A fundamental tool in our article is the nonhomogeneous Harnack inequality.

Theorem 2.2 (Harnack inequality; see e.g. [15]). Let $u \in W^{1, p}\left(B_{R}\right)$ be such that $u \geq 0$ a.e., and satisfy

$$
\operatorname{div} \mathcal{A}(X, \nabla u)=g(X), \quad \text { in } B_{R}
$$

in the distributional sense, with $g(X) \in L^{\infty}\left(B_{R}\right)$. Then, there exists a constant $C>0$ such that, for all $0<r \leq R$,

$$
\sup _{B_{r}} u \leq C\left\{\inf _{B_{r}} u+\left(r^{p}\|g\|_{L^{\infty}\left(B_{R}\right)}\right)^{1 /(p-1)}\right\} .
$$

Remark 2.3. Let $\Omega^{\prime} \subset \mathbb{R}^{n}$ be a bounded domain such that $B_{R} \subset \Omega^{\prime}$. In this article we will often regard the nonlinear term merely as a bounded source term, i.e.,

$$
m f(X) u^{m}(X)=: g(X)
$$

where $\|g\|_{L^{\infty}\left(B_{R}\right)} \leq C$ for a universal constant $C>0$. In particular, for $0 \leq r \leq 1$ we have

$$
\sup _{B_{r}} u \leq C\left\{\inf _{B_{r}} u+r^{p /(p-1)}\left(\|g\|_{L^{\infty}\left(B_{R}\right)}\right)^{1 /(p-1)}\right\} \leq C \inf _{B_{r}} u+C r,
$$

where $C=C\left(n, p, \lambda, \Lambda,\|g\|_{L^{\infty}\left(\Omega^{\prime}\right)}\right)>0$. 
To address Lipschitz regularity for a minimizer $u$ we will need the $C^{1, \alpha}$ regularity of $\mathcal{A}$-harmonic functions. Precisely, the result that will be useful is the following (see [18] for superior estimates at degenerate points):

Theorem $2.4\left(C^{1, \alpha}\right.$ regularity; see e.g. [19]). Let $\Omega \subset \mathbb{R}^{n}$ be a bounded open set and let $g \in L^{\infty}(\Omega)$ satisfying

$$
\operatorname{div}(\mathcal{A}(X, \nabla u))=g(X), \quad \text { in } \Omega
$$

in the distributional sense. Then, there exist constants $C>0$ and $0<\alpha<1$ that depend only on $\Omega^{\prime},\|g\|_{L^{\infty}\left(\Omega^{\prime}\right)}$, and universal constants, such that

$$
\|\nabla u\|_{L^{\infty}\left(\Omega^{\prime}\right)} \leq C \quad \text { and } \quad\|u\|_{C^{1, \alpha}\left(\Omega^{\prime}\right)} \leq C,
$$

where $\Omega^{\prime} \Subset \Omega$ is a subdomain.

Remark 2.5. We point out that Theorem 2.4 for $\Omega^{\prime}=B_{2 r}\left(X_{0}\right)$ gives

$$
\|\nabla u\|_{L^{\infty}\left(B_{r}\left(X_{0}\right)\right)} \leq C r^{\gamma-1} \text { and } \quad\|\nabla u\|_{C^{\alpha}\left(B_{r}\left(X_{0}\right)\right)} \leq C r^{\gamma-1-\alpha}
$$

for some universal constant $0<\gamma<1$. Also we shall make use of the gradient estimate:

$$
\|\nabla h\|_{L^{\infty}\left(B_{r / 2}\left(X_{0}\right)\right)} \leq \frac{C}{r^{n / p}}\|\nabla h\|_{L^{p}\left(B_{r}\left(X_{0}\right)\right)}
$$

for $\mathcal{A}$-harmonic functions, where $C>0$ is universal constant; see for instance [8], Proposition 3.3. Sharp estimates below Lipschitz can be found, for instance, in [17].

We shall also make use of the following technical lemma regarding convergence stability.

Lemma 2.6. Let $h_{j}: B_{1}(0) \rightarrow(0,2)$ be a sequence of function in $W^{1, p}\left(B_{1}(0)\right)$ such that

$$
\operatorname{div}\left(\mathcal{A}\left(W_{j}+c_{j} X, \nabla h_{j}\right)\right)=0 \quad \text { in } B_{1}(0),
$$

where $c_{j} \in \mathbb{R}^{n}$ and $W_{j} \in \Omega$ satisfy

$$
c_{j} \rightarrow 0, \quad W_{j} \rightarrow W_{0} \in \Omega \text { and } W_{j}+c_{j} X \in \Omega,
$$

for all $X \in B_{1}(0)$. Suppose

$$
h_{j} \rightarrow h_{0} \text { a.e. in } B^{\prime} \text {, }
$$

where $B^{\prime} \Subset B_{1}(0)$ is a ball. Then, we have, for all $B \Subset B^{\prime}$,

$$
\operatorname{div}\left(\mathcal{A}\left(W_{0}, \nabla h_{0}\right)\right)=0 \quad \text { in } B
$$


Proof. Since each function $h_{j}$ satisfies $0 \leq h_{j} \leq 2$ we conclude from the Caccioppoli inequality ([11], Chapter 3, Lemma 3.27) that there exists a constant $C>0$ that depends only on $B^{\prime}$ and universal constants such that

$$
\int_{B^{\prime}}\left|\nabla h_{j}\right|^{p} d X \leq C .
$$

Thus (see for instance [11], Theorem 1.32), $h_{0} \in W^{1, p}\left(B^{\prime}\right)$ and

$$
\nabla h_{j} \rightarrow \nabla h_{0} \quad \text { weakly in } L^{p}\left(B^{\prime}\right) \text {. }
$$

Consider $\zeta \in C_{0}^{\infty}\left(B^{\prime}\right)$ such that $\zeta=1$ in $B$ and $0 \leq \zeta \leq 1$. Take $\zeta\left(h_{0}-h_{j}\right)$ as a test function for the solution $h_{j}$. Then we obtain, for $Q_{j}:=W_{j}+c_{j} X$,

$$
\begin{gathered}
\left|\int_{B^{\prime}} \zeta\left\langle\mathcal{A}\left(Q_{j}, \nabla h_{j}\right), \nabla h_{j}-\nabla h_{0}\right\rangle d X\right|=\left|\int_{B^{\prime}}\left(h_{j}-h_{0}\right)\left\langle\mathcal{A}\left(Q_{j}, \nabla h_{j}\right), \nabla \zeta\right\rangle d X\right| \\
\leq C\left(\int_{B^{\prime}}\left|h_{j}-h_{0}\right|^{p}|\nabla \zeta|^{p}\right)^{1 / p} \cdot\left(\int_{B^{\prime}}\left|\nabla h_{j}\right|^{p}\right)^{(p-1) / p} \leq C\left(\int_{B^{\prime}}\left|h_{j}-h_{0}\right|^{p}\right)^{1 / p}
\end{gathered}
$$

where we use that (see [11], Chapter 5, Lemma 5.9)

$$
|\mathcal{A}(X, \xi)| \leq C(p, \lambda)|\xi|^{p-1}, \quad \text { for a.e. } X \in \Omega \text { and all } \xi \in \mathbb{R}^{n} \text {, }
$$

and (2.5) in the second inequality.

Thus, since $h_{j} \rightarrow h_{0}$ a.e in $B^{\prime}$, we conclude from the dominated convergence theorem that

$$
\lim _{j \rightarrow \infty} \int_{B^{\prime}} \zeta\left\langle\mathcal{A}\left(W_{j}+c_{j} X, \nabla h_{j}\right), \nabla h_{j}-\nabla h_{0}\right\rangle d X=0 .
$$

Using (2.6) we obtain

$$
\left|\int_{B^{\prime}} \zeta\left\langle\mathcal{A}\left(Q_{j}, \nabla h_{0}\right), \nabla h_{j}-\nabla h_{0}\right\rangle d X\right| \leq C(p, \lambda) \int_{B^{\prime}}\left|\nabla h_{0}\right|^{p-1}\left|\nabla h_{j}-\nabla h_{0}\right| d X .
$$

Thus, since $\nabla h_{0} \in L^{p /(p-1)}\left(B^{\prime}\right)$, we find

$$
\lim _{j \rightarrow \infty} \int_{B^{\prime}} \zeta\left\langle\mathcal{A}\left(W_{j}+c_{j} X, \nabla h_{0}\right), \nabla h_{j}-\nabla h_{0}\right\rangle d X=0 .
$$

By monotonicity of $\mathcal{A}$, we have

$$
\int_{B^{\prime}} \zeta\left\langle\mathcal{A}\left(W_{j}+c_{j} X, \nabla h_{j}\right)-\mathcal{A}\left(W_{j}+c_{j} X, \nabla h_{0}\right), \nabla h_{j}-\nabla h_{0}\right\rangle d X \geq 0
$$

Then, we find

$$
\lim _{j \rightarrow \infty} \int_{B}\left\langle\mathcal{A}\left(W_{j}+c_{j} X, \nabla h_{j}\right)-\mathcal{A}\left(W_{0}+c_{j} X, \nabla h_{0}\right), \nabla h_{j}-\nabla h_{0}\right\rangle d X=0 .
$$


Moreover, it follows from (G4) that

$$
\left|\int_{B}\left\langle\mathcal{A}\left(Q_{j}, \nabla h_{j}\right)-\mathcal{A}\left(W_{0}, \nabla h_{j}\right), \nabla h_{j}-\nabla h_{0}\right\rangle d X\right| \leq \frac{C_{\mathcal{A}}}{2} \int_{B}\left|\nabla h_{j}-\nabla h_{0}\right| d X,
$$

for $j$ so large that $\left(\left|W_{j}-W_{0}\right|+\left|c_{j}\right|\right)^{\delta} \leq 1 / 2$. Taking into account that $\chi_{B} \in$ $L^{p /(p-1)}(B)$ we obtain

$$
\lim _{j \rightarrow \infty} \int_{B}\left\langle\mathcal{A}\left(W_{j}+c_{j} X, \nabla h_{j}\right)-\mathcal{A}\left(W_{0}, \nabla h_{j}\right), \nabla h_{j}-\nabla h_{0}\right\rangle d X=0 .
$$

Analogously, we have

$$
\lim _{j \rightarrow \infty} \int_{B}\left\langle\mathcal{A}\left(W_{j}+c_{j} X, \nabla h_{0}\right)-\mathcal{A}\left(W_{0}, \nabla h_{0}\right), \nabla h_{j}-\nabla h_{0}\right\rangle d X=0 .
$$

Hence, we obtain

$$
\lim _{j \rightarrow \infty} \int_{B}\left\langle\mathcal{A}\left(W_{0}, \nabla h_{j}\right)-\mathcal{A}\left(W_{0}, \nabla h_{j}\right), \nabla h_{j}-\nabla h_{0}\right\rangle d X=0
$$

and by Lemma 3.73 in [11] we find

$$
\mathcal{A}\left(W_{0}, \nabla h_{j}\right) \rightarrow \mathcal{A}\left(W_{0}, \nabla h_{0}\right),
$$

weakly in $L^{p /(p-1)}(B)$. Finally, if $\eta \in C_{0}^{\infty}(B)$ we have

$$
\begin{aligned}
0 & =\int_{B}\left\langle\mathcal{A}\left(Q_{j}, \nabla h_{j}\right), \nabla \eta\right\rangle d X \\
& =\int_{B}\left\langle\mathcal{A}\left(Q_{j}, \nabla h_{j}\right)-\mathcal{A}\left(W_{0}, \nabla h_{j}\right), \nabla \eta\right\rangle d X+\int_{B}\left\langle\mathcal{A}\left(W_{0}, \nabla h_{j}\right), \nabla \eta\right\rangle d X
\end{aligned}
$$

Taking $j \rightarrow \infty$ we obtain

$$
\int_{B}\left\langle\mathcal{A}\left(W_{0}, \nabla h_{0}\right), \nabla \eta\right\rangle d X=0
$$

and the proof is complete.

\section{Existence and continuity of minimizers}

In this section we show the discontinuous optimization problem (1.1) has at least one minimizer. Uniqueness is known to fail even in simpler models. In the sequel we obtain a universal modulus of continuity for such a minimum.

Theorem 3.1. There exists a minimizer $u \in W_{\phi}^{1, p}$ of the functional (1.1). Furthermore $u \geq 0$ in $\Omega$. 
Proof. Let us label

$$
I_{0}:=\min \left\{\int_{\Omega} F(X, v, \nabla v) d X: v \in W_{\phi}^{1, p}(\Omega)\right\} .
$$

To begin, we show that $I_{0}>-\infty$. Indeed, for any $v \in W_{\phi}^{1, p}(\Omega)$, by the Poincaré, Young $(1<p / m)$ and Hölder inequalities, there exist universal constants $c, C>0$ such that

$$
c\|v\|_{L^{p}}^{p}-c\|\phi\|_{L^{p}}^{p}-\lambda\|\nabla \phi\|_{L^{p}}^{p} \leq \lambda\|\nabla v\|_{L^{p}}^{p}
$$

and

$$
-c\|v\|_{L^{p}}^{p}-C \leq-C\|v\|_{L^{p}}^{m} \leq-K\|v\|_{L^{m}}^{m} .
$$

Combining (3.1) and (3.2) we obtain

$$
-C-c\|\phi\|_{L^{p}}^{p}-\lambda\|\nabla \phi\|_{L^{p}}^{p} \leq \lambda\|\nabla v\|_{L^{p}}^{p}-K\|v\|_{L^{m}}^{m},
$$

which yields

$$
-C-c\|\phi\|_{L^{p}}^{p}-\lambda\|\nabla \phi\|_{L^{p}}^{p} \leq \int_{\Omega}\left(\lambda|\nabla v|^{p}-K|v|^{m}+\kappa \chi_{\{v>0\}}\right) d X .
$$

Finally, from (G2) and (g1) we find

$$
\int_{\Omega}\left(\lambda|\nabla v|^{p}-K|v|^{m}+\kappa \chi_{\{v>0\}}\right) d X \leq \int_{\Omega} F(X, v, \nabla v) d X .
$$

Let $v_{j} \in W_{\phi}^{1, p}(\Omega)$ be a minimizing sequence. We can suppose for $j \gg 1$, that

$$
\int_{\Omega} F\left(X, v_{j}, \nabla v_{j}\right) d X \leq I_{0}+1
$$

From (3.4) and the Hölder inequality we obtain

$$
\int_{\Omega}\left|\nabla v_{j}\right|^{p} d X \leq \frac{K}{\lambda}\left\|v_{j}\right\|_{L^{m}}^{m}+\frac{I_{0}}{\lambda}+\frac{1}{\lambda} \leq C\left\|v_{j}\right\|_{L^{p}}^{m}+\frac{I_{0}}{\lambda}+C .
$$

By the Poincaré inequality we have

$$
c_{1}\left\|v_{j}\right\|_{L^{p}}^{m} \leq C_{2}\left(\left\|\nabla v_{j}\right\|_{L^{p}}^{m}+\|\nabla \phi\|_{L^{p}}^{m}+\|\phi\|_{L^{p}}^{m}\right) .
$$

Also we have

$$
c_{3}\left\|\nabla v_{j}\right\|_{L^{p}}^{m} \leq C_{4}+\frac{1}{2}\left\|\nabla v_{j}\right\|_{L^{p}}^{p}
$$

Combining (3.5), (3.6) and (3.7) we obtain

$$
\int_{\Omega}\left|\nabla v_{j}\right|^{p} d X \leq C_{5}\left(\|\nabla \phi\|_{L^{p}}^{m}+\|\phi\|_{L^{p}}^{m}\right)+\frac{I_{0}}{\lambda}+C_{6} .
$$


Thus, using the Poincaré inequality once more, we conclude that $\left\{v_{j}-\phi\right\}$ is a bounded sequence in $W_{0}^{1, p}(\Omega)$. By weak compactness, there is a function $u \in$ $W_{\phi}^{1, p}(\Omega)$ such that, passing to a subsequence, if necessary,

$$
v_{j} \rightarrow u \text { weakly in } W^{1, p}(\Omega), \quad v_{j} \rightarrow u \text { in } L^{p}(\Omega) \quad v_{j} \rightarrow u \text { a.e. in } \Omega \text {. }
$$

It now follows from the lower semicontinuity of $G$ (see, for instance, [11], Chapter 5) that

$$
\int_{\Omega} G(X, \nabla u) d X \leq \liminf _{j \rightarrow \infty} \int_{\Omega} G\left(X, \nabla v_{j}\right) d X .
$$

Condition (g1) and pointwise convergence give

$$
\int_{\Omega} g(X, u) d X \leq \liminf _{j \rightarrow \infty} \int_{\Omega} g\left(X, v_{j}\right) d X
$$

In conclusion,

$$
\int_{\Omega} F(X, u, \nabla u) d X \leq \liminf _{j \rightarrow \infty} \int_{\Omega} F\left(X, v_{j}, \nabla v_{j}\right) d X
$$

which proves the existence of a minimizer.

We turn our attention to proving the nonnegativity of $u$. To begin, we note that

$$
\chi_{\{\max (u, 0)>0\}} \leq \chi_{\{u>0\}}
$$

Thus,

$$
\begin{aligned}
& \int_{\Omega} g(X, \max (u, 0))-g(X, u) d X \\
& \quad=\int_{\Omega} f\left(\left(u^{+}\right)^{m}-\left(u^{+}\right)^{m}\right) d X+\int_{\Omega} Q\left(\chi_{\{\max (u, 0)>0\}}-\chi_{\{u>0\}}\right) d X \leq 0 .
\end{aligned}
$$

Then, by minimality of $u$ and (3.9) we obtain

$$
\begin{aligned}
0 & \leq \int_{\Omega} F(X, \max (u, 0), \nabla(\max (u, 0)))-F(X, u, \nabla u) d X \\
& =\int_{\Omega} G(X, \nabla(\max (u, 0)))-G(X, \nabla u) d X+\int_{\Omega} g(X, \max (u, 0))-g(X, u) d X \\
& \leq-\int_{\{u<0\}} G(X, \nabla u) d X .
\end{aligned}
$$

From (G2) we can write

$$
0 \geq \int_{\{u \leq 0\}} G(X, \nabla u) d X \geq \lambda \int_{\{u<0\}}|\nabla u|^{p} d X=\lambda \int_{\Omega}|\nabla(\min (u, 0))|^{p} d X,
$$

and the nonnegativity of $u$ follows since the boundary data $\phi$ is nonnegative. 
Remark 3.2. As previously stated, for condition (g1), throughout the whole paper we shall work under the range $1 \leq m<p$. Such a constraint is needed merely for the existence of minima of the functional $\mathfrak{F}$. Also, by inequalities (3.1), (3.5) and (3.6), it is possible to show existence of minimizer provided $K$ is small enough. In addition, we can obtain critical points of the functional $\mathfrak{F}$, in the range $p<m \leq p^{*}($ see $[14])$ where $p^{*}:=n p /(n-p)$.

Lemma 3.3. Let $u$ be a minimizer of (1.1). There exists a universal constant $M>0$ such that $\|u\|_{L^{\infty}(\Omega)} \leq M$.

Proof. Define

$$
j_{0}:=\left\lceil\sup _{\partial \Omega} \phi\right\rceil
$$

that is, the smallest natural number above $\sup _{\partial \Omega} \phi$. For each $j \geq j_{0}$ we define the truncated function $u_{j}: \Omega \rightarrow \mathbb{R}$ by

$$
u_{j}= \begin{cases}j, & \text { if } u>j \\ u, & \text { if } u \leq j\end{cases}
$$

Clearly, by the choice of $j_{0}, u_{j} \in W_{\phi}^{1, p}(\Omega)$ and

$$
\left\{u_{j}>0\right\}=\{u>0\} .
$$

If we define $A_{j}:=\{u>j\}$, we have, for each $j>j_{0}$

$$
u=u_{j} \text { in } A_{j}^{c} \text { and } u_{j}=j \text { in } A_{j} .
$$

Thus, by minimality of $u$ and (G2), there holds

$$
\begin{aligned}
\lambda \int_{A_{j}}|\nabla u|^{p} d X & \leq \int_{A_{j}} G(X, \nabla u)=\int_{\Omega} G(X, \nabla u)-G\left(X, \nabla u_{j}\right) d X \\
& \leq \int_{\Omega} f\left(u_{j}^{m}-u^{m}\right) d X=\int_{A_{j}} f\left(u^{m}-j^{m}\right) d X
\end{aligned}
$$

Taking into account the elementary inequality

$$
u^{m}=(u-j+j)^{m} \leq 2^{m}\left[(u-j)^{m}+j^{m}\right],
$$

we obtain

$$
\begin{aligned}
\int_{A_{j}}|f|\left(u^{m}-j^{m}\right) d X & \leq C \int_{A_{j}}|f|(u-j)^{m} d X+C j^{m} \mathcal{L}^{n}\left(A_{j}\right) \\
& =C \int_{A_{j}}|f|\left[(u-j)^{+}\right]^{m} d X+C j^{m} \mathcal{L}^{n}\left(A_{j}\right) .
\end{aligned}
$$

From the range of truncation we consider, it follows that $(u-j)^{+} \in W_{0}^{1, p}(\Omega)$. Hence, applying the Hölder inequality and Gagliardo-Nirenberg inequalities (see Chapter 7 of $[10])$, we find

$$
\int_{A_{j}}|f|\left[(u-j)^{+}\right]^{m} d X \leq K\left[\mathcal{L}^{n}\left(A_{j}\right)\right]^{1-m / p^{*}}\|\nabla u\|_{L^{p}\left(A_{j}\right)}^{m} .
$$


The Young inequality then gives

$$
K\left[\mathcal{L}^{n}\left(A_{j}\right)\right]^{1-m / p^{*}}\|\nabla u\|_{L^{p}\left(A_{j}\right)}^{m} \leq C\left[\mathcal{L}^{n}\left(A_{j}\right)\right]^{\frac{p}{p-m}-\frac{p m}{p^{*}(p-m)}}+\frac{\lambda}{2 C}\|\nabla u\|_{L^{p}\left(A_{j}\right)}^{p}
$$

Since

$$
1-\left[\frac{p}{p-m}-\frac{p m}{p^{*}(p-m)}\right]=-\frac{p^{2} m}{n p(p-m)}
$$

we find

$$
\left[\mathcal{L}^{n}\left(A_{j}\right)\right]^{\frac{p}{p-m}-\frac{p m}{p^{*}(p-m)}} \leq C \mathcal{L}^{n}\left(A_{j}\right) .
$$

Then, combining (3.10), (3.11), and (3.12), we obtain

$$
\int_{A_{j}}|\nabla u|^{p} d X \leq C j^{m} \mathcal{L}^{n}\left(A_{j}\right)=C j^{m}\left[\mathcal{L}^{n}\left(A_{j}\right)\right]^{1-p / n+p / n} .
$$

Moreover, for each $j>j_{0}$ we have

$$
\begin{aligned}
\left\|(u-j)^{+}\right\|_{L^{1}\left(A_{j}\right)} & =\int_{A_{j}}(u-j) d X \leq \int_{A_{j}}\left(u-j_{0}\right) d X \leq \int_{A_{j_{0}}}\left(u-j_{0}\right) d X \\
& =\left\|\left(u-j_{0}\right)^{+}\right\|_{L^{1}\left(A_{j_{0}}\right)} \leq\|u\|_{L^{1}\left(A_{j_{0}}\right)}+C\left(\phi, \mathcal{L}^{n}(\Omega)\right) .
\end{aligned}
$$

We also have (see (3.1) and (3.8) replacing $I_{0}$ by $\left.\int_{\Omega} F(X, \phi, \nabla \phi) d X\right)$,

$$
\|u\|_{L^{1}\left(A_{j_{0}}\right)} \leq\left[\mathcal{L}^{n}\left(A_{j_{0}}\right)\right]^{(p-1) / p}\|u\|_{L^{p}\left(A_{j_{0}}\right)} \leq\left[\mathcal{L}^{n}(\Omega)\right]^{(p-1) / p}\|u\|_{L^{p}(\Omega)} \leq C .
$$

Taking $\gamma=C, \varepsilon=p / n$ and $\alpha=m$ in Lemma 2.1 we obtain the boundedness of $u$.

Remark 3.4. A consequence of the $L^{\infty}$ estimates for a minimizer $u$ of the functional $\mathfrak{F}$ is universal control on $u$ in $W^{1, p}(\Omega)$. In fact, we have

$$
\lambda^{-1} \int_{\Omega}|\nabla u|^{p} d X \leq \mathfrak{F}(\phi)-\int_{\Omega} f(X)\left(u^{+}\right)^{m}+Q \chi_{\{u>0\}} d X \leq \mathfrak{F}(\phi)+C \leq C,
$$

where $C>0$ is a universal constant. In conclusion,

$$
\|u\|_{W^{1, p}(\Omega)} \leq C
$$

Lemma 3.5 (Caccioppoli type inequality). Let $u$ be a minimizer of the functional (1.1). If $B_{r}\left(X_{0}\right) \subset \Omega$ is a ball such that $0<2 r \leq \operatorname{dist}\left(X_{0}, \partial \Omega\right)$ then we have the estimate

$$
\int_{B_{r}\left(X_{0}\right)}|\nabla u|^{p} d X \leq C r^{n-p}
$$

where $C>0$ is a universal constant. 
Proof. Let $\zeta \in C_{0}^{\infty}(\Omega)$ be a nonnegative function. Given $\varepsilon>0$, by the minimality and nonnegativity of $u$, we have

$$
\begin{aligned}
0 \leq & \frac{1}{\varepsilon} \int_{\Omega} G(X, \nabla(u-\varepsilon \zeta))-G(X, \nabla u) d X \\
& +\frac{1}{\varepsilon} \int_{\Omega} f(X)\left[\left((u-\varepsilon \zeta)^{+}\right)^{m}-u^{m}\right] d X+\frac{1}{\varepsilon} \int_{\Omega} Q\left(\chi_{\{u-\varepsilon \zeta>0\}}-\chi_{\{u>0\}}\right) d X \\
\leq & -\int_{\Omega}\langle\mathcal{A}(X, \nabla(u-\varepsilon \zeta)), \nabla \zeta\rangle d X+\frac{1}{\varepsilon} \int_{\Omega} f(X)\left[\left((u-\varepsilon \zeta)^{+}\right)^{m}-u^{m}\right] d X .
\end{aligned}
$$

Also, for $\phi:=(u-\varepsilon \zeta)^{+}$, we have

$$
\begin{aligned}
\frac{1}{\varepsilon} \int_{\Omega} & f(X)\left[\phi^{m}-u^{m}\right] d X \\
\quad= & \frac{1}{\varepsilon} \int_{\{u>0\}} f(X)\left[\phi^{m}-u^{m}\right] d X+\frac{1}{\varepsilon} \int_{\{u=0\}} f(X)\left[(-\varepsilon \zeta)^{+}\right]^{m} d X \\
\quad= & \int_{\{u>0\}} f(X) \frac{\left[\phi^{m}-u^{m}\right]}{\varepsilon} d X .
\end{aligned}
$$

Taking $\varepsilon \rightarrow 0$ we obtain

$$
-\int_{\Omega}\langle\mathcal{A}(X, \nabla u), \nabla \zeta\rangle d X-\int_{\Omega}\left(m f(X) u^{m-1} \chi_{\{u>0\}}\right) \zeta d X \geq 0 .
$$

If $B_{r}:=B_{r}\left(X_{0}\right)$ let $\eta \in C_{0}^{\infty}\left(B_{r}\right)$ be a nonnegative function such that

$$
\eta= \begin{cases}1, & \text { in } B_{r}, \\ 0, & \text { in } B_{2 r}^{c},\end{cases}
$$

and

$$
\|\nabla \eta\|_{L^{\infty}(\Omega)} \leq \frac{1}{2 r}
$$

From (G3) we obtain

$$
\langle\mathcal{A}(X, \xi), \xi\rangle=p G(X, \xi),
$$

for almost every $X \in \Omega$ and all $\xi \in \mathbb{R}^{n}$. Moreover, we have (see [11], Chapter 5 , Lemma 5.9)

$$
|\mathcal{A}(X, \xi)| \leq C(p, \lambda)|\xi|^{p-1}
$$

for a.e. $X \in \Omega$ and all $\xi \in \mathbb{R}^{n}$.

Then, taking $\zeta=\eta^{p} u$ in inequality (3.15) and using (3.18) and (3.19) we find

$$
\begin{aligned}
0 \leq & \int_{\Omega}\left\langle\mathcal{A}(X, \nabla u),-\eta^{p} \nabla u-p \eta^{p-1} u \nabla \eta\right\rangle d X-\int_{\Omega}\left(m f(X) u^{m-1} \chi_{\{u>0\}}\right) \eta^{p} u d X \\
\leq & -C(p, \lambda) \int_{\Omega}|\nabla u|^{p} \eta^{p} d X+C(p, \lambda) \int_{\Omega} u|\nabla \eta||\nabla u|^{p-1} \eta^{p-1} d X \\
& +m M^{m} K \int_{\Omega} \eta^{p} d X .
\end{aligned}
$$


Thus, we find

$$
\begin{aligned}
C(p, \lambda) \int_{\Omega}|\nabla u|^{p} \eta^{p} d X \leq & C(p, \lambda) \int_{\Omega} u^{p}|\nabla \eta|^{p} d X+\frac{C(p, \lambda)}{2} \int_{\Omega}|\nabla u|^{p} \eta^{p} d X \\
& +C(m, M, K) \int_{\Omega} \eta^{p} d X
\end{aligned}
$$

where we use the Young inequality. Hence, we obtain

$$
\int_{\Omega}|\nabla u|^{p} \eta^{p} d X \leq C(p, \lambda) \int_{\Omega} u^{p}|\nabla \eta|^{p} d X+C(m, M, K, p, \lambda) \int_{\Omega} \eta^{p} d X,
$$

and taking into account the conditions (3.16), (3.17) and the boundedness of $u$ (Lemma 3.3) we obtain

$$
\int_{B_{r}}|\nabla u|^{p} d X \leq C\left[\frac{C}{r^{p}} \mathcal{L}^{n}\left(B_{2 r}\right)+C \mathcal{L}^{n}\left(B_{2 r}\right)\right] \leq C\left(r^{n-p}+r^{n}\right) \leq C r^{n-p},
$$

and the lemma is proved.

Even though the functional is discontinuous, it is possible to prove that minimizers are universally continuous. The delicate question of optimal regularity will be addressed in the next section.

Theorem 3.6. Let $u$ be a minimizer of (1.1). There exists a universal constant $\beta \in(0,1)$ such that $u \in C_{\mathrm{loc}}^{0, \beta}(\Omega)$.

Proof. Let $h$ be the solution of boundary value problem

$$
\left\{\begin{array}{rlrl}
\operatorname{div}(\mathcal{A}(X, \nabla h))=0 & & \text { in } B . \\
h & =u & & \text { on } \partial B
\end{array}\right.
$$

where $B \Subset \Omega$ is a fixed ball. By the minimality of $u,(\mathrm{~g} 1)$ and the mean value theorem we have

$$
\begin{aligned}
\int_{B} G(X, & \nabla u)-G(X, \nabla h) d X \leq \int_{B} g(X, h)-g(X, u) d X \\
& =\int_{B} f\left(h^{m}-u^{m}\right) d X+\int_{B} Q\left(\chi_{\{h>0\}}-\chi_{\{u>0\}}\right) d X \\
& \leq m K M^{m-1} \int_{B}|u-h| d X+\kappa^{-1} \mathcal{L}^{n}(\{u=0\} \cap B) .
\end{aligned}
$$

We have used that an $\mathcal{A}$-harmonic function with nonnegative boundary values is positive and $0 \leq u, h \leq M$ (see [11], Chapter 3, Proposition 3.24). Thus, using (3.18) and monotonicity, see for instance, Lemma 3.2 in [16], we obtain

$$
\int_{B} G(X, \nabla u)-G(X, \nabla h) d X \geq c \int_{B}|\nabla(u-h)|^{p} d X
$$


where $c=c(n, p, G)$ is a positive constant. The Young and Poincaré inequalities together yield

$$
m K M^{m-1} \int_{B}|u-h| d X \leq \frac{c}{2} \int_{B}|\nabla(u-h)|^{p} d X+C \mathcal{L}^{n}(B) .
$$

Thus, if $B$ is a ball of radius $r>0$, it follows from (3.20), (3.21) and (3.22) that

$$
\|\nabla(u-h)\|_{L^{p}(B)} \leq C r^{n / p} .
$$

Now we will estimate $\|\nabla h\|_{L^{p}(B)}$. If $B=B_{r}\left(X_{0}\right)$ for some $X_{0} \in \Omega$ and $r>0$ we have (see (2.4) and (2.2) in section 2)

$$
\|\nabla h\|_{L^{\infty}\left(B_{r / 2}\left(X_{0}\right)\right)} \leq \frac{C}{r^{n / p}}\|\nabla h\|_{L^{p}(B)} \leq \frac{C}{r}
$$

where we use Lemma 3.5 in the last inequality.

Let $0<r \leq r_{0}(\varepsilon)$ with $r_{0}^{\varepsilon} \leq 1 / 2$ for a constant $\varepsilon>0$ to be chosen. By the inequalities (3.23) and (3.24) we estimate

$\|\nabla u\|_{L^{p}\left(B_{r^{1}+\varepsilon}\right)} \leq\|\nabla(u-h)\|_{L^{p}\left(B_{r^{1+\varepsilon}}\right)}+\|\nabla h\|_{L^{p}\left(B_{r^{1+\varepsilon}}\right)}$

$$
\leq\|\nabla(u-h)\|_{L^{p}\left(B_{r}\right)}+C r^{\frac{(1+\varepsilon) n}{p}}\|\nabla h\|_{L^{\infty}\left(B_{r / 2}\right)} \leq C\left(r^{\frac{n}{p}}+r^{\frac{(1+\varepsilon) n}{p}-1}\right) .
$$

Taking $\tau=r^{1+\varepsilon}$ we obtain

$$
\|\nabla u\|_{L^{p}\left(B_{\delta}\right)} \leq C \tau^{n / p-(1-\theta)},
$$

with $0<\theta=\theta(\varepsilon)<1$ if $\varepsilon=\varepsilon(n, p)>0$ is small.

Hence, from Morrey's theorem there is a constant $\beta=\beta(n, p)>0$ such that $u \in C_{\mathrm{loc}}^{0, \beta}(\Omega)$.

Remark 3.7. In Theorem 3.6 we can consider the cases $p=n$ and $p>n$ (the later is trivial). Moreover, If $h$ is as in Theorem 3.6 we have $\|h\|_{L^{\infty}\left(B_{r / 2}\left(X_{0}\right)\right)} \leq M$. Thus, if $G$ satisfies the assumption of Theorem 2.4 (see Remark 2.4), we obtain

$$
\|\nabla h\|_{L^{\infty}\left(B_{r / 2}\left(X_{0}\right)\right)} \leq \frac{C}{r^{1-\gamma}} \leq \frac{C \mathcal{L}^{n}(\Omega)^{\gamma}}{r} \leq \frac{C_{1}}{r}
$$

and the arguments of Theorem 3.6 remain valid. Here we do not use (2.2) and Lemma 3.5 in the proof of Theorem 3.6.

At this stage of the program, an important consequence of Theorem 3.6 is the fact that the positivity set, $\{u>0\}$, of $u$ is open. Next theorem gives the Euler-Lagrange equations satisfied in this set.

Theorem 3.8. Let $u$ be a minimizer of (1.1). Within the open set $\{u>0\}, u$ satisfies

$$
\operatorname{div}(\mathcal{A}(X, \nabla u))=m f(X) u^{m-1}
$$

in the distributional sense. 
Proof. For fixed $\zeta \in C_{0}^{\infty}(\{u>0\})$, there is $0<\varepsilon_{0} \ll 1$, so small that

$$
\{u+\varepsilon \zeta>0\}=\{u>0\},
$$

for all $0<\varepsilon \leq \varepsilon_{0}$. We can write, for $v_{\varepsilon}:=u+\varepsilon \zeta$,

$$
\begin{aligned}
& \frac{1}{\varepsilon} \int_{\{u>0\}}\left(F\left(X, v_{\varepsilon}, \nabla v_{\varepsilon}\right)-F(X, u, \nabla u)\right) d X \\
& =\frac{1}{\varepsilon} \int_{\{u>0\}} G\left(X, \nabla v_{\varepsilon}\right)-G(X, \nabla u)+\int_{\{u>0\}} f(X) \frac{v_{\varepsilon}^{m}-u^{m}}{\varepsilon} .
\end{aligned}
$$

Taking $\varepsilon \rightarrow 0$, and using the minimality of $u$, we obtain

$$
0=\int_{\{u>0\}}\langle\mathcal{A}(X, \nabla u), \nabla \zeta\rangle d X+\int_{\{u>0\}} m f(X) u^{m-1} \zeta d X
$$

and the result follows.

\section{Upper and lower gradient bounds}

In the previous section we have shown that minimizers are $C^{0, \beta}$ continuous in $\Omega$, for some unknown $\beta<1$. From the discontinuity of the functional $\mathfrak{F}$ along the free-surface it is also possible to check that minimizers are not $C^{1}$-regular across the zero level surface $\partial\{u>0\}$. Thus the optimal regularity one can expect for $u$ is Lipschitz continuity. This is the content of the next theorem.

Theorem 4.1. Given a subdomain $\Omega^{\prime} \Subset \Omega$, there exists a constant $C>0$ that depends only on $\Omega^{\prime}$ and universal constants, such that

$$
\|\nabla u\|_{L^{\infty}\left(\Omega^{\prime}\right)} \leq C
$$

Proof. Suppose, with the aim of obtaining a contradiction, that there exists a sequence of points $X_{j} \in \Omega^{\prime} \cap\{u>0\}$ such that

$$
X_{j} \rightarrow \partial\{u>0\} \quad \text { and } \quad \frac{u\left(X_{j}\right)}{\operatorname{dist}\left(X_{j}, \partial\{u>0\}\right)} \nearrow \infty
$$

We write

$$
U_{j}:=u\left(X_{j}\right) \quad \text { and } \quad d_{j}:=\operatorname{dist}\left(X_{j}, \partial\{u>0\}\right) .
$$

For each $j$, let $Y_{j} \in \partial\{u>0\}$ be such that

$$
d_{j}=\left|X_{j}-Y_{j}\right|
$$

Recall we have proven in Theorem 3.8 that

$$
\operatorname{div}(\mathcal{A}(X, \nabla u))=m f(X) u^{m-1} \quad \text { in }\{u>0\} .
$$


Thus, by the Harnack inequality (Theorem 2.2), universal boundedness of $u$, and (g1), there exists a constant $c>0$ (see Remark 2.3) that depends only on $\Omega^{\prime}$ and universal constants, such that

$$
d_{j}+\inf _{\bar{B}_{3 d_{j} / 4}\left(X_{j}\right)} u \geq c U_{j}
$$

In turn, we have

$$
\sup _{\bar{B}_{d_{j} / 4}\left(Y_{j}\right)} u \geq c U_{j}-d_{j}
$$

Consider the set

$$
A_{j}:=\left\{Z \in B_{d_{j}}\left(Y_{j}\right): \operatorname{dist}(Z, \partial\{u>0\}) \leq \frac{1}{3} \operatorname{dist}\left(Z, \partial B_{d_{j}}\left(Y_{j}\right)\right)\right\} .
$$

First we claim that $B_{d_{j} / 4}\left(Y_{j}\right) \subset A_{j}$. In fact, if $\left|Z-Y_{j}\right| \leq d_{j} / 4$, then

$$
\frac{1}{3} \operatorname{dist}\left(Z, \partial B_{d_{j}}\left(Y_{j}\right)\right) \geq \frac{1}{3} \frac{3 d_{j}}{4}=\frac{d_{j}}{4} \geq \operatorname{dist}(Z, \partial\{u>0\}) .
$$

Thus,

$$
M_{j}:=\sup _{Z \in A_{j}} \operatorname{dist}\left(Z, \partial B_{d_{j}}\left(Y_{j}\right)\right) u(Z)=\operatorname{dist}\left(Z_{j}, \partial B_{d_{j}}\left(Y_{j}\right)\right) u\left(Z_{j}\right) \geq \frac{3 d_{j}}{4} \sup _{\bar{B}_{d_{j} / 4}\left(Y_{j}\right)} u .
$$

Therefore,

$$
u\left(Z_{j}\right) \geq \frac{d_{j}}{\operatorname{dist}\left(Z_{j}, \partial B_{d_{j}}\left(Y_{j}\right)\right)} \frac{3}{4} \sup _{\bar{B}_{d_{j} / 4}\left(Y_{j}\right)} u \geq \frac{3}{4} \sup _{\bar{B}_{d_{j} / 4}\left(Y_{j}\right)} u .
$$

Hence, using (4.2) we have

$$
u\left(Z_{j}\right) \geq \frac{3}{4}\left(c U_{j}-d_{j}\right) .
$$

For each $j$, let $W_{j} \in \partial\{u>0\}$ be such that

$$
r_{j}:=\left|Z_{j}-W_{j}\right|=\operatorname{dist}\left(Z_{j}, \partial\{u>0\}\right) \leq \frac{1}{3} \operatorname{dist}\left(Z_{j}, \partial B_{d_{j}}\left(Y_{j}\right)\right) .
$$

Using (4.5) we conclude that

$$
r_{j} \leq \frac{1}{3}\left(d_{j}-\left|Z_{j}-Y_{j}\right|\right) \leq \frac{1}{3}\left(d_{j}-r_{j}\right) .
$$

That is,

$$
\frac{d_{j}}{r_{j}} \geq 4
$$

From (4.4) and (4.7) we have, for $j$ so large that

$$
\frac{U_{j}}{d_{j}} \geq \frac{1}{c}, \quad\left(c \frac{U_{j}}{d_{j}}-1 \geq 0\right)
$$


the lower estimate

$$
\frac{u\left(Z_{j}\right)}{r_{j}} \geq \frac{1}{r_{j}} \frac{3}{4}\left(c U_{j}-d_{j}\right)=\frac{3 d_{j}}{4 r_{j}}\left(c \frac{U_{j}}{d_{j}}-1\right) \geq 4 \frac{3}{4}\left(c \frac{U_{j}}{d_{j}}-1\right) .
$$

We have proven that

$$
\frac{u\left(Z_{j}\right)}{r_{j}} \rightarrow \infty
$$

If $X \in B_{2 r_{j}}\left(W_{j}\right)$ we obtain (see (4.5))

$$
\left|X-Y_{j}\right| \leq\left|X-W_{j}\right|+\left|W_{j}-Z_{j}\right|+\left|Z_{j}-Y_{j}\right| \leq 2 r_{j}+r_{j}+\left|Z_{j}-Y_{j}\right| \leq d_{j} .
$$

Thus, $B_{2 r_{j}}\left(W_{j}\right) \subset B_{d_{j}}\left(Y_{j}\right)$. Also we have

$$
\operatorname{dist}(X, \partial\{u>0\}) \leq \frac{r_{j}}{2},
$$

for all $X \in B_{r_{j} / 2}\left(W_{j}\right)$. The triangular inequality and (4.5) then yield

$$
\begin{gathered}
\operatorname{dist}\left(X, \partial B_{d_{j}}\left(Y_{j}\right)\right) \geq \operatorname{dist}\left(Z_{j}, \partial B_{d_{j}}\left(Y_{j}\right)\right)-\left|Z_{j}-X\right| \geq \operatorname{dist}\left(Z_{j}, \partial B_{d_{j}}\left(Y_{j}\right)\right)-\frac{3 r_{j}}{2} \\
\geq \operatorname{dist}\left(Z_{j}, \partial B_{d_{j}}\left(Y_{j}\right)\right)-\frac{1}{2} \operatorname{dist}\left(Z_{j}, \partial B_{d_{j}}\left(Y_{j}\right)\right)=\frac{1}{2} \operatorname{dist}\left(Z_{j}, \partial B_{d_{j}}\left(Y_{j}\right)\right) .
\end{gathered}
$$

We conclude that $B_{r_{j} / 2}\left(W_{j}\right) \subset A_{j}$ and

$$
u\left(Z_{j}\right) \geq \frac{M_{j}}{\operatorname{dist}\left(Z_{j}, \partial B_{d_{j}}\left(Y_{j}\right)\right)} \geq \frac{\operatorname{dist}\left(X, \partial B_{d_{j}}\left(Y_{j}\right)\right) u(X)}{\operatorname{dist}\left(Z_{j}, \partial B_{d_{j}}\left(Y_{j}\right)\right)} \geq \frac{1}{2} u(X),
$$

for all $X \in B_{r_{j} / 2}\left(W_{j}\right)$. From the preceding inequality we obtain

$$
\sup _{B_{r_{j} / 2}\left(W_{j}\right)} u \leq 2 u\left(Z_{j}\right)
$$

Since $B_{r_{j}}\left(Z_{j}\right) \subset\{u>0\}$, by the Harnack inequality there exists a universal constant $c^{\prime}>0$ that depends only on $\Omega^{\prime}$ and universal constants such that

$$
\inf _{\bar{B}_{3 r_{j} / 4}\left(Z_{j}\right)} u \geq c^{\prime} u\left(Z_{j}\right)-r_{j} .
$$

Therefore, we conclude,

$$
\sup _{\bar{B}_{r_{j} / 4}\left(W_{j}\right)} u \geq c^{\prime} u\left(Z_{j}\right)-r_{j}
$$

and using (4.9) we find (for $j$ sufficiently large)

$$
\sup _{\bar{B}_{r_{j} / 4}\left(W_{j}\right)} \frac{u}{u\left(Z_{j}\right)} \geq \frac{c^{\prime}}{2}
$$


For each $j$, consider the normalized function $u_{j}: B_{1}(0) \rightarrow(0,2)$, defined by

$$
u_{j}(X):=\frac{u\left(W_{j}+\frac{1}{2} r_{j} X\right)}{u\left(Z_{j}\right)} .
$$

Notice that from (4.10), and (4.11), we have (for $j$ sufficiently large)

$$
\max _{B_{1}(0)} u_{j} \leq 2, \quad \max _{B_{1}(0)} u_{j} \geq \frac{c^{\prime}}{2}, \quad u_{j}(0)=0 .
$$

Let $h$ be the $\mathcal{A}$-harmonic function in $B_{r_{j} / 2}\left(W_{j}\right)$ equal to $u$ on the boundary. By (3.20), as in Theorem 3.6, we have

$\int_{B_{r_{j} / 2}\left(W_{j}\right)}\langle\mathcal{A}(X, \nabla u), \nabla u\rangle-\langle\mathcal{A}(X, \nabla h), \nabla h\rangle d X \leq K \int_{B_{r_{j} / 2}\left(W_{j}\right)}\left|u^{m}-h^{m}\right| d X+C r_{j}^{n}$.

Analogously, for each $j$ sufficiently large, consider the normalized function $h_{j}: B_{1}(0) \rightarrow(0,2)$, defined by

$$
h_{j}(X):=\frac{h\left(W_{j}+\frac{1}{2} r_{j} X\right)}{u\left(Z_{j}\right)} .
$$

It is easy to see that

$$
\left\{\begin{aligned}
\operatorname{div}\left(\mathcal{A}\left(W_{j}+\frac{1}{2} r_{j} X, \nabla h_{j}\right)\right) & =0 & & \text { in } B_{1}(0) \\
h_{j} & =u_{j} & & \text { on } \partial B_{1}(0)
\end{aligned}\right.
$$

Also, from the normalization,

$$
\nabla u_{j}(X)=\frac{r_{j}}{2 u\left(Z_{j}\right)} \nabla u\left(W_{j}+\frac{1}{2} r_{j} X\right), \quad \nabla h_{j}(X)=\frac{r_{j}}{2 u\left(Z_{j}\right)} \nabla h\left(W_{j}+\frac{1}{2} r_{j} X\right),
$$

for all $X \in B_{1}(0)$. By a change of variables and (G3) we obtain

$$
\begin{aligned}
\int_{B_{r_{j} / 2}\left(W_{j}\right)}\left|u^{m}-h^{m}\right| d X & \leq u^{m}\left(Z_{j}\right) C(m)\left(\frac{r_{j}}{2}\right)^{n} \int_{B_{1}(0)}\left|u_{j}-h_{j}\right| d X \\
& \leq C(n, m) u^{m}\left(Z_{j}\right)\left(\frac{r_{j}}{2}\right)^{n} .
\end{aligned}
$$

Similarly,

$$
\int_{B_{r_{j} / 2}\left(W_{j}\right)}\langle\mathcal{A}(X, \nabla u), \nabla u\rangle d X=\varsigma_{j} \cdot \int_{B_{1}(0)}\left\langle\mathcal{A}\left(W_{j}+\frac{r_{j}}{2} X, \nabla u_{j}\right), \nabla u_{j}\right\rangle d X
$$

where

$$
\varsigma_{j}:=\left(\frac{r_{j}}{2 u\left(Z_{j}\right)}\right)^{-p}\left(\frac{r_{j}}{2}\right)^{n}
$$


We conclude that

$$
\begin{aligned}
c \int_{B_{1}(0)}\left|\nabla\left(u_{j}-h_{j}\right)\right|^{p} d X \leq & \int_{B_{1}(0)}\left\langle\mathcal{A}\left(W_{j}+\frac{r_{j}}{2} X, \nabla u_{j}\right), \nabla u_{j} d X\right\rangle \\
& -\int_{B_{1}(0)}\left\langle\mathcal{A}\left(W_{j}+\frac{r_{j}}{2} X, \nabla h_{j}\right), \nabla h_{j}\right\rangle d X \\
\leq & l_{j} \rightarrow 0 .
\end{aligned}
$$

where

$$
l_{j}:=C r_{j}^{m}\left(\frac{r_{j}}{u\left(Z_{j}\right)}\right)^{p-m}=C u^{m}\left(Z_{j}\right)\left(\frac{r_{j}}{u\left(Z_{j}\right)}\right)^{p} \leq C\left(\frac{r_{j}}{u\left(Z_{j}\right)}\right)^{p}
$$

Moreover, $u_{j}$ is a minimizer of the functional

$$
\mathcal{F}_{j}(v):=\int_{B_{1}} G_{j}(X, \nabla v)+f_{j}(X)\left(v^{+}\right)^{m}+Q_{j}(X) \chi_{\{v>0\}} d X,
$$

where

$$
\begin{aligned}
G_{j}(X, \xi) & :=G\left(W_{j}+\frac{r_{j}}{2} X, \xi\right), \quad \text { for all } X \in B_{1}, \xi \in \mathbb{R}^{n}, \\
f_{j}(X) & :=2^{m} u^{m}\left(Z_{j}\right)\left(\frac{r_{j}}{2 u\left(Z_{j}\right)}\right)^{p} f\left(W_{j}+\frac{r_{j}}{2} X\right), \quad \text { for all } X \in B_{1}
\end{aligned}
$$

and

$$
Q_{j}(X):=\left(\frac{r_{j}}{2 u\left(Z_{j}\right)}\right)^{p} Q\left(W_{j}+\frac{r_{j}}{2} X\right), \quad \text { for all } X \in B_{1} .
$$

Since the functional $\mathcal{F}_{j}$ satisfies the structural conditions (G1), (G2), (G3), (G4), and (g1) and $0 \leq u_{j} \leq 2$ in $B_{1}$ we conclude, as in the proof of Theorem 3.6, that $u_{j}$ and $h_{j}$ are uniform Hölder continuous in $B_{8 / 9}(0)$. Thus, up to a subsequence,

$$
u_{j} \rightarrow u_{0} \text { and } h_{j} \rightarrow h_{0},
$$

uniformly in $\overline{B_{4 / 9}}(0)$, and (see Lemma 2.6 and Remark 3.4)

$$
\nabla u_{j} \rightarrow \nabla u_{0} \text { and } \nabla h_{j} \rightarrow \nabla h_{0}
$$

weakly in $L^{p}\left(B_{4 / 9}(0)\right)$. Passing to the limit in (4.16), we find (see Lemma 2.6)

$$
\operatorname{div}\left(\mathcal{A}\left(W_{0}, \nabla h_{0}\right)\right)=0 \quad \text { in } B_{2 / 9}(0),
$$

where, up to a subsequence, $W_{j} \rightarrow W_{0} \in \partial\{u>0\}$. From (4.18) we find

$$
\nabla\left(u_{j}-h_{j}\right) \rightarrow 0 \text { weakly in } L^{p}\left(B_{2 / 9}(0)\right) .
$$

Then, we obtain $\nabla\left(u_{0}-h_{0}\right)=0$ in $L^{p}\left(B_{4 / 9}(0)\right)$. Thus, $u_{0}=h_{0}+c$, for some $c \in \mathbb{R}$, and it solves the elliptic PDE

$$
\operatorname{div}\left(\mathcal{A}\left(W_{0}, \nabla u_{0}\right)\right)=0 \quad \text { in } B_{2 / 9}(0) .
$$

Therefore, since $u_{0}(0)=0$ and $u_{0} \geq 0$, we obtain, by the strong maximum principle, that $u_{0} \equiv 0$ in $B_{2 / 9}(0)$, which contradicts (4.13). Theorem 4.1 is proven. 
The optimal regularity estimate on $u$ established in Theorem 4.1 implies that $u$ grows at most linearly away from the free surface $\partial\{u>0\}$. From energy considerations, we will show next that minimizers grow precisely linearly.

Theorem 4.2. Given a subdomain $\Omega^{\prime} \Subset \Omega$, there exist constants $c_{1}>0$ and $r_{1}>0$ that depend only on $\Omega^{\prime}$ and universal constants, such that if $X_{0} \in \partial\{u>0\} \cap \Omega^{\prime}$, $0<r \leq r_{1}$, then

$$
\sup _{X \in B_{r}\left(X_{0}\right)} u(X) \geq c_{1} r
$$

Proof. Given a point $X_{0} \in \partial\{u>0\} \cap \Omega^{\prime}$, define

$$
v(X)=\frac{u\left(X_{0}+r X\right)}{r}, \quad \forall X \in B_{1}(0) .
$$

Since $u$ is Lipschitz continuous we have

$$
\|v\|_{L^{\infty}\left(B_{1}(0)\right)} \leq C .
$$

Let $h_{r}$ be the universal barrier given by

$$
\left\{\begin{aligned}
\operatorname{div}\left(\mathcal{A}\left(X_{0}+r X, \nabla h_{r}\right)\right)=0 & \text { in } B_{1}(0) \backslash B_{1 / 2}(0) \\
h_{r}=1 & \text { on } \partial B_{1}(0) \\
h_{r}=0 & \text { in } \overline{B_{1 / 2}(0)}
\end{aligned}\right.
$$

Define the test function $\xi$ in $B_{1}(0)$ by

$$
\xi(X)=\min \left\{v(X), h_{r}(X) \sup _{B_{1}(0)} v\right\}
$$

Using (4.21) we have

$$
0 \leq \xi \leq v \leq C
$$

By the minimality of $v$ in $B_{1}(0)$, if we define $\Pi:=\left\{h_{r} \sup _{B_{1}\left(X_{0}\right)} u<v\right\}$ and

$$
\Phi:=\int_{\Pi} G\left(X_{0}+r X, \nabla \xi\right)-G\left(X_{0}+r X, \nabla v\right) d X
$$

we can estimate

$$
\begin{aligned}
\Phi= & \int_{B_{1}(0)} G\left(X_{0}+r X, \nabla \xi\right)-G\left(X_{0}+r X, \nabla v\right) d X \\
\geq & \int_{B_{1}(0)} g\left(X_{0}+r X, r v\right)-g\left(X_{0}+r X, r \xi\right) d X \\
= & r^{m} \int_{\Pi} f\left(X_{0}+r X\right)\left(v^{m}-\xi^{m}\right) d X \\
& \quad+\int_{B_{1}(0)} Q\left(X_{0}+r X\right)\left(\chi_{\{v>0\}}-\chi_{\{\xi>0\}}\right) d X .
\end{aligned}
$$


Also we have

$$
\begin{aligned}
& r^{m} \int_{\Pi}\left|f\left(X_{0}+r X\right)\left(v^{m}-\xi^{m}\right)\right| d X \leq r^{m} K m C^{m-1} \int_{\Pi}|v-\xi| d X \\
& \quad \leq r^{m} C \int_{\Pi}|v-\xi|^{p} d X+r^{m} C \leq r^{m} C \int_{\Pi}|\nabla(v-\xi)|^{p} d X+r^{m} C \\
& \quad \leq r^{m} C \int_{\Pi}|\nabla v|^{p} d X+r^{m} C \int_{\Pi}|\nabla \xi|^{p} d X+r^{m} C .
\end{aligned}
$$

Similarly we estimate

$$
\begin{aligned}
\int_{B_{1}(0)} & Q\left(X_{0}+r X\right)\left(\chi_{\{v>0\}}-\chi_{\{\xi>0\}}\right) d X \\
= & \int_{B_{1}(0)} Q\left(X_{0}+r X\right)\left(1-\chi_{\{\xi>0\}}\right) d X \\
= & \int_{B_{1}(0)} Q\left(X_{0}+r X\right) \chi_{\{\xi=0\}} d X \geq \epsilon \mathcal{L}^{n}(\{\xi=0\}) \geq \epsilon \mathcal{L}^{n}\left(B_{1 / 2}(0)\right),
\end{aligned}
$$

where $C>0$ is a universal constant. Taking into account (G2) and (G3), we obtain

$$
\begin{aligned}
\int_{\Pi} G & \left(X_{0}+r X, \nabla \xi\right)-G\left(X_{0}+r X, \nabla v\right) d X \\
& \leq \lambda^{-1} \int_{\Pi}\left|\sup _{B_{1}(0)} v \nabla h_{r}\right|^{p} d X-\lambda \int_{\Pi}|\nabla v|^{p} d X \\
& \leq C\left(\sup _{B_{1}(0)} v\right)^{p} \int_{B_{1}(0)}\left|\nabla h_{r}\right|^{p} d X-\lambda \int_{\Pi}|\nabla v|^{p} d X,
\end{aligned}
$$

for a universal $C>0$. Also by the $C^{1, \alpha}$ estimates for $h_{r}$, we have

$$
C\left(\sup _{B_{1}(0)} v\right)^{p} \int_{B_{1}(0)}\left|\nabla h_{r}\right|^{p} d X \leq C\left(\sup _{B_{1}(0)} v\right)^{p}
$$

for a universal constant $C>0$. Substituting inequalities (4.23), (4.24) and (4.25) into (4.22) and taking $r$ sufficiently small, we obtain

$$
C\left(\sup _{B_{1}(0)} v\right)^{p} \geq\left(\lambda-r^{m} C\right) \int_{\Pi}|\nabla v|^{p} d X+c \mathcal{L}^{n}\left(B_{1 / 2}(0)\right) \geq c \mathcal{L}^{n}\left(B_{1 / 2}(0)\right),
$$

for universal constants $c>0$ and $C>0$. Therefore, $\sup _{B_{1}(0)} v \geq c$, and strong nondegeneracy is proven.

Corollary 4.3. Given a subdomain $\Omega^{\prime} \Subset \Omega$, there exist constants $c, d_{0}>0$ that depend only on $\Omega^{\prime}$ and universal constants, such that if $X \in\{u>0\} \cap \Omega^{\prime}$ and $\operatorname{dist}(X, \partial\{u>0\}) \leq d_{0}$, then

$$
u(X) \geq c \cdot \operatorname{dist}(X, \partial\{u>0\}) .
$$

We emphasize that the technique employed in proof of Theorem 4.2 is a generalization of the ones used in [1]. 


\section{Hausdorff estimates on the free boundary}

In this section we turn out attention to fine Hausdorff estimates on the free surface $\partial\{u>0\}$.

Theorem 5.1. Given a subdomain $\Omega^{\prime} \Subset \Omega$ and $Z \in \partial\{u>0\} \cap \Omega^{\prime}$, there exist constants $r_{0}>0$ and $0<\varsigma<1$ that depend only on $\Omega^{\prime}$ and universal constants, such that,

$$
\varsigma \omega_{n} r^{n} \leq \mathcal{L}^{n}\left(B_{r}(Z) \cap\{u>0\}\right) \leq(1-\varsigma) \omega_{n} r^{n},
$$

for all $0 \leq r \leq r_{0}$.

Proof. Let $X_{0} \in \bar{B}_{r / 4}(Z)$ be a maximum point of $u$, i.e.,

$$
u\left(X_{0}\right)=\sup _{X \in B_{r / 4}(Z)} u(X)
$$

By the strong nondegeneracy property $\left(0<r \leq r_{0}\right.$ with $r_{0}=r_{1}$ and $r_{1}>0$ as in Theorem 4.2) we obtain

$$
u\left(X_{0}\right) \geq c r>0,
$$

where $c>0$ is a universal constant. By the Lipschitz continuity of $u$ there exists a universal constant $C>0$ such that

$$
u(X) \geq u\left(X_{0}\right)-C\left|X-X_{0}\right| \geq \frac{c r}{2}>0, \quad \forall X \in B_{\frac{c}{2 C} r}\left(X_{0}\right),
$$

with $C \geq c$. Hence,

$$
B_{\frac{c}{2 C} r}\left(X_{0}\right) \subset B_{r}(Z) \cap\{u>0\},
$$

and the lower bound in (5.1) follows.

Now we prove the upper bound. We argue by contradiction, i.e., let us assume that there exists a sequence of positive real numbers $r_{j}$ with $r_{j} \searrow 0$ as $j \rightarrow \infty$ and

$$
\frac{\mathcal{L}^{n}\left(B_{r_{j}}(Z) \cap\{u=0\}\right)}{r_{j}^{n}} \rightarrow 0 .
$$

We define the sequence $u_{j}: B_{1}(0) \rightarrow \mathbb{R}$ by

$$
u_{j}(X):=\frac{u\left(Z+r_{j} X\right)}{r_{j}}
$$

Let $h_{j}$ be the solution to

$$
\left\{\begin{aligned}
\operatorname{div}\left(\mathcal{A}\left(Z+r_{j} X, \nabla h_{j}\right)\right) & =0 & & \text { in } B_{1}(0) \\
h_{j} & =u_{j} & & \text { on } \partial B_{1}(0) .
\end{aligned}\right.
$$


Notice that by Lipschitz continuity of $u$, both $u_{j}$ and $h_{j}$ are bounded. Estimates (3.20) and (3.21) from the proof of Theorem 3.6 give after renormalization,

$$
\begin{aligned}
& \int_{B_{1}(0)}\left|\nabla\left(h_{j}-u_{j}\right)\right|^{p} d X \leq r_{j}^{m} \int_{B_{1}(0)}\left|h_{j}^{m}-u_{j}^{m}\right| d X+C \frac{\mathcal{L}^{n}\left(B_{r_{j}}(Z) \cap\{u=0\}\right)}{r_{j}^{n}} \\
& \leq r_{j}^{m} C \int_{B_{1}(0)}\left|h_{j}-u_{j}\right| d X+C \frac{\mathcal{L}^{n}\left(B_{r_{j}}(Z) \cap\{u=0\}\right)}{r_{j}^{n}} \\
& \quad \leq r_{j}^{m} C \int_{B_{1}(0)}\left|h_{j}-u_{j}\right|^{p} d X+r_{j}^{m} C+C \frac{\mathcal{L}^{n}\left(B_{r_{j}}(Z) \cap\{u=0\}\right)}{r_{j}^{n}} \\
& \quad \leq r_{j}^{m} C \int_{B_{1}(0)}\left|\nabla\left(h_{j}-u_{j}\right)\right|^{p} d X+r_{j}^{m} C+C \frac{\mathcal{L}^{n}\left(B_{r_{j}}(Z) \cap\{u=0\}\right)}{r_{j}^{n}},
\end{aligned}
$$

where $C>0$ is a universal constant. Hence, for $j$ sufficiently large we have

$$
\int_{B_{1}(0)}\left|\nabla\left(h_{j}-u_{j}\right)(X)\right|^{p} d X \leq r_{j}^{m} C+C \frac{\mathcal{L}^{n}\left(B_{r_{j}}(Z) \cap\{u=0\}\right)}{r_{j}^{n}} .
$$

Moreover, by the Lipschitz regularity of $u$ and the $C^{1, \alpha}$ elliptic estimate we can assume that

$$
u_{j} \rightarrow u_{0} \text { and } h_{j} \rightarrow h_{0}
$$

uniformly in $B_{4 / 5}(0)$. Since $h_{j}$ is the solution to problem (5.4) we obtain

$$
\operatorname{div}\left(\mathcal{A}\left(Z, \nabla h_{0}(Y)\right)\right)=0, \quad \text { in } B_{1 / 2}(0) .
$$

It follows from (5.2) and (5.5) that

$$
u_{0}=h_{0}+c, \text { in } B_{1 / 2}(0) .
$$

where $c$ is a constant. Then,

$$
\operatorname{div}\left(\mathcal{A}\left(Z, \nabla u_{0}(Y)\right)\right)=0, \quad \text { in } B_{1 / 2}(0) .
$$

Since $u_{0} \geq 0$ and $u_{0}(0)=0$, by the strong maximum principle, we obtain $u_{0} \equiv 0$ in $B_{1 / 2}(0)$. But this contradicts the strong nondegeneracy property, shown in Theorem 4.2 .

Theorem 3.8 gives the Euler-Lagrange equation $u$ satisfies within its set of positivity. To further investigate the behavior of $u$ along the free boundary, we need to obtain the equation $u$ satisfies across the free surface of discontinuity of the functional, $\partial\{u>0\}$.

Lemma 5.2. Let $u$ be a minimizer of the functional (1.1), then

$$
\operatorname{div}(\mathcal{A}(X, \nabla u))-m f(X) u^{m-1} \chi_{\{u>0\}} \geq 0, \quad \text { in } \Omega
$$

in the sense of distribution. In particular it defines a Radon measure

$$
\Lambda:=\operatorname{div}(\mathcal{A}(X, \nabla u))-m f(X) u^{m-1} \chi_{\{u>0\}}
$$

Furthermore, the support of $\Lambda$ is contained in $\partial\{u>0\}$. 
Proof. By Lemma 3.5 we have

$$
-\int_{\Omega}\langle\mathcal{A}(X, \nabla u), \nabla \zeta\rangle d X-\int_{\Omega}\left(m f(X) u^{m-1} \chi_{\{u>0\}}\right) \zeta d X \geq 0,
$$

for all nonnegative functions $\zeta \in C_{0}^{\infty}(\Omega)$. Moreover, as in Theorem 3.8,

$$
\operatorname{div} \mathcal{A}(X, \nabla u)=m f(X) u^{m-1} \quad \text { in }\{u>0\} .
$$

Hence, the measure $\Lambda$ defined by

$$
\int_{\Omega} \zeta d \Lambda:=-\int_{\Omega}\langle\mathcal{A}(X, \nabla u), \nabla \zeta\rangle d X-\int_{\Omega}\left(m f(X) u^{m-1} \chi_{\{u>0\}}\right) \zeta d X,
$$

is a nonnegative Radon measure with support on $\Omega \cap \partial\{u>0\}$.

With the aid of the measure $\Lambda$, we can establish fine upper and lower control on the $\mathcal{H}^{n-1}$ Hausdorff measure of the free boundary, which ultimately yields important geometric measure theoretic information about $\partial\{u>0\}$.

Theorem 5.3. The set $\{u>0\}$ has locally finite perimeter and for fixed $\Omega^{\prime} \Subset \Omega$ there exist constants $\underline{c}$ and $\bar{C}$ that depend only on $\Omega^{\prime}$ and universal constants such that

$$
\underline{c} r^{n-1} \leq \mathcal{H}^{n-1}\left(\partial\{u>0\} \cap B_{r}(Z)\right) \leq \bar{C} r^{n-1}
$$

for any ball $B_{r}(Z) \subset \Omega^{\prime}$ centered at a free boundary point, $Z \in \partial\{u>0\}$. In particular,

$$
\mathcal{H}^{n-1}\left(\partial\{u>0\} \backslash \partial_{\text {red }}\{u>0\}\right)=0 .
$$

Proof. Through a suitable approximation scheme, using test function $0 \leq \zeta_{k} \leq 1$, $\zeta_{k}$ with $\zeta_{k} \rightarrow \chi_{B_{r}(Z)}$, we have (for almost $r>0$ )

$$
\begin{aligned}
\int_{B_{r}(Z)} d \Lambda & =\int_{\partial B_{r}(Z)}\langle\mathcal{A}(X, \nabla u), \nu\rangle d S_{X}-\int_{B_{r}(Z)} m f(X) u^{m-1} \chi_{\{u>0\}} d X \\
& \leq C\|\nabla u\|_{L^{\infty}\left(\Omega^{\prime}\right)}^{p-1} r^{n-1}+C r^{n-1} \leq C r^{n-1}
\end{aligned}
$$

where $C>0$ is a constant that depend only on $\Omega^{\prime}$ and universal constants. This proves the upper bound in (5.7).

To check the lower bound, let us assume, aiming at a contradiction, that there exists a sequence of positive real numbers $r_{j}$ such that $r_{j} \searrow 0$ as $j \rightarrow \infty$ and

$$
\frac{\mathcal{H}^{n-1}\left(\partial\{u>0\} \cap B_{r_{j}}(Z)\right)}{r_{j}^{n-1}} \rightarrow 0 .
$$

With the notation used in Theorem 5.1 and Lemma 5.2, we obtain the sequence of nonnegative measures $\Lambda_{j}$ in $B_{4 / 5}(0)$, defined by

$$
\Lambda_{j}:=\left[\operatorname{div}\left(\mathcal{A}\left(Z+r_{j} X, \nabla u_{j}\right)\right)-r_{j}^{m} m f\left(Z+r_{j} X\right) \chi_{\left\{u_{j}>0\right\}} u_{j}^{m-1}\right] d X .
$$


By compactness we can assume that $\Lambda_{j} \rightarrow \Lambda_{0}$ in the sense of measures. Moreover, using (5.9) we have

$$
\Lambda_{j} \rightarrow 0
$$

In the sequel we will show that

$$
\Lambda_{0}:=\operatorname{div}\left(\mathcal{A}\left(Z, \nabla u_{0}\right)\right)
$$

From the uniform positive density property we know $\mathcal{L}^{n}\left(\partial\left\{u_{0}>0\right\}\right)=0$. Thus, we only need to verify (5.12) for balls $B$ contained entirely in $\left\{u_{0}=0\right\}$ and in $\left\{u_{0}>0\right\}$. Let $B \subset\left\{u_{0}>0\right\}$. Define

$$
A_{j}(X):=\mathcal{A}\left(Z+r_{j} X, \nabla u_{j}\right), \quad \forall X \in B_{4 / 5}(0) .
$$

By the Lipschitz regularity of $u$ and (G2) we have

$$
\left|A_{j}\right| \leq C(n, \Lambda)\left|\nabla u_{j}\right|^{p-1} \leq C .
$$

Thus, we may extract a subsequence (that we denote by $A_{j}$ ) such that

$$
A_{j} \rightarrow A_{0} \quad \text { weak- } \star \text { in } L^{\infty}\left(B_{4 / 5}\right) .
$$

Furthermore, $u_{j}$ converges in the $C^{1, \alpha}$ topology to $u_{0}$ in $B$ (see Section 2, Theorem 2.4 and Remark 5.5 below). Hence,

$$
A_{j} \rightarrow \mathcal{A} q\left(Z, \nabla u_{0}\right) \quad \text { weak- } \star \text { in } L^{\infty}(B) .
$$

Also we have

$$
\left|\int_{B} r_{j}^{m} m f\left(Z+r_{j} X\right) \chi_{\left\{u_{j}>0\right\}} u_{j}^{m-1} d X\right| \leq r_{j}^{m} C \mathcal{L}^{n}(B) \rightarrow 0 .
$$

Hence, for $B \subset\left\{u_{0}>0\right\}$, indeed (5.12) does hold. Now suppose $B \subset\left\{u_{0}=0\right\}$. Clearly,

$$
\left[\operatorname{div}\left(\mathcal{A} q\left(Z, \nabla u_{0}\right)\right)\right](B)=0 .
$$

On the other hand, if $B_{k}$ is a sequence of balls such that $B_{k} \nearrow B$ then for some $j_{k} \in \mathbb{N}$ we have

$$
u_{j} \equiv 0 \quad \text { in } B_{k} \text { for all } j>j_{k} .
$$

Indeed, let $\tilde{B} \subset B$. If there were a subsequence $u_{j_{k}}$ satisfying $u_{j_{k}} \neq 0$ in $\tilde{B}$ then, by the strong nondegeneracy property (Theorem 4.2), there must exist points $P_{k_{j}} \in \tilde{B}$ such that

$$
u_{j_{k}}\left(P_{k_{j}}\right) \geq c>0 .
$$

Passing to another subsequence we can assume $P_{k_{j}} \rightarrow P \in \tilde{B}$. Since $u_{j_{k}} \rightarrow u_{0}$ uniformly we obtain $u_{0}(P)>0$ which is a contradiction.

Thus, from (5.14) we obtain

$$
\Lambda_{j}(B) \rightarrow 0 .
$$


Therefore, (5.12) holds for any $B \subset\left\{u_{0}=0\right\}$, and combining (5.11) and (5.12) we find

$$
\operatorname{div}\left(\mathcal{A}\left(Z, \nabla u_{0}\right)\right)=0 \quad \text { in } B_{4 / 5}(0) .
$$

However, as before, this contradicts the nondegeneracy of $u_{0}$ established in Theorem 4.2.

Theorem 5.4 (Representation). Let $u$ be a minimizer of (1.1). Then

$$
\operatorname{div}(\mathcal{A}(X, \nabla u))-m f(X) u^{m-1} \chi_{\{u>0\}}=\tilde{Q} \mathcal{H}^{n-1}\left\lfloor\partial_{\text {red }}\{u>0\},\right.
$$

in the sense of measures, for some Borel function $\tilde{Q}$. Moreover, $\tilde{Q}$ is bounded away from zero and infinity. That is for a universal constant $C>0$ there holds

$$
C^{-1} \leq \tilde{Q} \leq C
$$

In particular, the free boundary $\partial\{u>0\}$ is a set of finite perimeter.

Proof. This follows from Theorem 5.3 and standard arguments (see Theorem 4.5 in [1]).

Remark 5.5. Let $u$ be a minimizer of (1.1) in $\Omega$ and let $B_{r_{j}}\left(X_{j}\right) \subset \Omega$ be a sequence of balls with $r_{j} \rightarrow 0, X_{j} \rightarrow X_{0} \in \Omega$, and $u\left(X_{j}\right)=0$. Consider the blow-up sequence

$$
u_{j}(X)=\frac{1}{r_{j}} u\left(X_{j}+r_{j} X\right)
$$

Since the $u_{j}$ are uniformly Lipschitz continuous, for a subsequence,

$$
\begin{aligned}
& u_{j} \rightarrow u_{0} \quad \text { in } C_{\text {loc }}^{\alpha}\left(\mathbb{R}^{n}\right) \text { for every } 0<\alpha<1, \\
& \nabla u_{j} \rightarrow \nabla u_{0} \quad \text { weak- } \star \text { in } L_{\text {loc }}^{\infty}\left(\mathbb{R}^{n}\right), \\
& \partial\left\{u_{j}>0\right\} \rightarrow \partial\left\{u_{0}>0\right\} \quad \text { locally in the Hausdorff distance, } \\
& \chi_{\left\{u_{j}>0\right\}} \rightarrow \chi_{\left\{u_{0}>0\right\}} \quad \text { in } L_{\text {loc }}^{1}\left(\mathbb{R}^{n}\right) .
\end{aligned}
$$

Moreover, by the classical truncation argument (see for instance, [2]),

$$
\nabla u_{j} \rightarrow \nabla u_{0} \quad \text { a.e. }
$$

Also, by Theorem 3.8, assertions (5.16)-(5.20) and $C^{1, \alpha}$ convergence within the positive set, we obtain

$$
\begin{aligned}
-\int_{\left\{u_{0}>0\right\}}\left\langle\mathcal{A}\left(X_{0}, \nabla u_{0}\right), \nabla \zeta\right\rangle d X & =-\lim _{j \rightarrow \infty} \int_{\left\{u_{0}>0\right\}}\left\langle\mathcal{A}\left(X_{j}+r_{j} X, \nabla u_{j}\right), \nabla \zeta\right\rangle d X \\
& =\lim _{j \rightarrow \infty} r_{j}^{m} \int_{\left\{u_{j}>0\right\}} m f\left(X_{j}+r_{j} X\right) u_{j}^{m-1} \zeta d X=0,
\end{aligned}
$$


for all $\zeta \in C_{0}^{\infty}\left(\left\{u_{0}>0\right\}\right)$. In fact,

$$
\left|r_{j}^{m} \int_{\left\{u_{j}>0\right\}} m f\left(X_{j}+r_{j} X\right) u_{j}^{m} \zeta d X\right| \leq r_{j}^{m} C
$$

for all $\zeta \in C_{0}^{\infty}\left(\left\{u_{0}>0\right\}\right)$. Hence,

$$
\operatorname{div}\left(\mathcal{A}\left(X_{0}, \nabla u_{0}\right)\right)=0 \quad \text { in }\left\{u_{0}>0\right\} .
$$

We are in position to obtain the blown-up minimization problem.

Lemma 5.6. If $u\left(X_{j}\right)=0, X_{j} \rightarrow X_{0} \in \Omega$, then any blow-up limit $u_{0}$ with respect to $B_{r_{j}}\left(X_{j}\right)$ is minimizer of the functional

$$
\mathfrak{F}_{0}(v):=\int_{B_{1}(0)} G\left(X_{0}, \nabla v\right)+Q\left(X_{0}\right) \chi_{\{v>0\}} d X .
$$

Proof. Set $D=B_{1}(0)$. Take any $v, v-u_{0} \in H_{0}^{1}(D), \eta \in C_{0}^{\infty}(D), 0 \leq \eta \leq 1$. Consider

$$
v_{j}:=v+(1-\eta)\left(u_{j}-u_{0}\right) \quad \text { and } \quad Q_{j}(X):=Q\left(X_{j}+r_{j} X\right), \quad \forall X \in D .
$$

We will write

$$
\mathfrak{F}_{j}(v):=\int_{B_{1}(0)} G\left(X_{j}+r_{j} X, \nabla v\right)+r_{j}^{m} f\left(X_{j}+r_{j} X\right)\left(v^{+}\right)^{m}+Q_{j}(X) \chi_{\{v>0\}} d X .
$$

Since $v_{j}=u_{j}$ in $\partial D$ and $u_{j}$ is a local minimum we have, for large $j$,

$$
\mathfrak{F}_{j}\left(u_{j}\right) \leq \mathfrak{F}_{j}\left(v_{j}\right)
$$

From the fact that $\left|\nabla u_{j}\right| \leq C$ and $\nabla u_{j} \rightarrow \nabla u_{0}$ a.e., we conclude

$$
\int_{D} G\left(X_{j}+r_{j} X, \nabla u_{j}\right) d X \rightarrow \int_{D} G\left(X_{0}, \nabla u_{0}\right) d X
$$

Similarly

$$
\int_{D} G\left(X_{j}+r_{j} X, \nabla v_{j}\right) d X \rightarrow \int_{D} G\left(X_{0}, \nabla v_{0}\right) d X
$$

Moreover, we have

$$
\left|\int_{D} r_{j}^{m} f\left(X_{j}+r_{j} X\right)\left(u_{j}^{m}-\left(v_{j}^{+}\right)^{m}\right) d X\right| \leq r_{j}^{m} C \int_{D}\left(\left|u_{j}\right|^{p^{*}}+\left|v_{j}\right|^{p^{*}}\right) d X,
$$

where, as before, $p^{*}:=n p /(n-p)$. Since there exists a universal constant $C>0$ such that $\left\|u_{j}\right\|_{L^{\infty}} \leq C$ (and $\Omega$ is bounded) we have

$$
\left\|u_{j}\right\|_{L^{p^{*}}} \leq C .
$$


By definition of $v_{j}$

$$
\left\|v_{j}\right\|_{L^{p^{*}}} \leq C\|v\|_{L^{p^{*}}}+C\left(\left\|u_{j}\right\|_{L^{p^{*}}}+\left\|u_{0}\right\|_{L^{p *}}\right) .
$$

Therefore,

$$
\left|\int_{D} r_{j}^{m} f\left(X_{j}+r_{j} X\right) u_{j}^{m}-r_{j}^{m} f\left(X_{j}+r_{j} X\right)\left(v_{j}^{+}\right)^{m} d X\right| \rightarrow 0 .
$$

Also, we have

$$
\left|\int_{D}\left(Q_{j}-Q\left(X_{0}\right)\right)\left(\chi_{\left\{u_{j}>0\right\}}-\chi_{\left\{v_{j}>0\right\}}\right) d X\right| \leq 2 \int_{D}\left|Q_{j}-Q\left(X_{0}\right)\right| d X
$$

and using the continuity of the function $Q$ we obtain

$$
\int_{D}\left(Q_{j}-Q\left(X_{0}\right)\right)\left(\chi_{\left\{u_{j}>0\right\}}-\chi_{\left\{v_{j}>0\right\}}\right) d X \rightarrow 0 .
$$

Finally,

$$
\chi_{\left\{v_{j}>0\right\}} \leq \chi_{\{v>0\}}+\chi_{\{\eta<1\}}
$$

and (see (5.19))

$$
\int_{D} \chi_{\left\{u_{j}>0\right\}} d X \rightarrow \int_{D} \chi_{\left\{u_{0}>0\right\}} d X .
$$

Then it follows from (5.22) that

$$
\begin{aligned}
\int_{D} G\left(X_{0}, \nabla u_{0}\right)+Q\left(X_{0}\right) \chi_{\left\{u_{0}>0\right\}} d X \leq & \int_{D} G\left(X_{0}, \nabla v\right) \\
& +\left(\chi_{\{v>0\}}+\chi_{\{\eta<1\}}\right) Q\left(X_{0}\right) d X .
\end{aligned}
$$

Taking $\eta \rightarrow 1$ finishes up the proof.

As a consequence, we can classify blow-ups at points in the reduced free boundary.

Theorem 5.7. For almost every $X_{0} \in \partial_{\text {red }}\{u>0\}$ we have

$$
u(X)=\alpha\left(X_{0}\right)\left\langle X-X_{0}, \nu\left(X_{0}\right)\right\rangle^{+}+o\left(\left|X-X_{0}\right|\right),
$$

for any $X \in\{u>0\}$ near $X_{0}$, where $\nu\left(X_{0}\right)$ is the measure theoretic normal vector to $\partial_{\text {red }}\{u>0\}$ at $X_{0}$ and

$$
\alpha\left(X_{0}\right)=\left(\frac{\tilde{Q}\left(X_{0}\right)}{\left\langle\mathcal{A}\left(X_{0}, \nu\left(X_{0}\right)\right), \nu\left(X_{0}\right)\right\rangle}\right)^{1 /(p-1)} .
$$

Proof. From 4.5.6 (2), 2.9.8, and 2.9.9 of [9], applied to $\mathcal{H}^{n-1}$ on $\partial\{u>0\}$ and the Vitali relation

$$
\left\{\left(X, B_{r}(X)\right): X \in \partial\{u>0\}, B_{r}(X) \Subset \Omega\right\},
$$


for almost every $X_{0} \in \partial_{\text {red }}\{u>0\}$ we have the condition

$$
\theta^{\star n-1}\left(\mathcal{H}^{n-1}\left\lfloor\partial\{u>0\}, X_{0}\right) \leq 1\right.
$$

and

$$
f_{B_{r}\left(X_{0}\right) \cap \partial\{u>0\}}\left|\tilde{Q}-\tilde{Q}\left(X_{0}\right)\right| d \mathcal{H}^{n-1}=o\left(r^{n-1}\right)
$$

for $r \rightarrow 0$, where the symbol in (5.25) represents the average. Recalling the notation used in Remark 5.5 we have, from standard geometric measure theory arguments together with nondegeneracy and assertions (5.16)-(5.20),

$$
\begin{aligned}
& u_{0} \equiv 0 \quad \text { in }\left\{X \in \mathbb{R}^{n}:\left\langle X, \nu\left(X_{0}\right)\right\rangle<0\right\} \\
\text { and } & \left\{u_{0}>0\right\}=\left\{X \in \mathbb{R}^{n}:\left\langle X, \nu\left(X_{0}\right)\right\rangle>0\right\} .
\end{aligned}
$$

Also we have

$$
\operatorname{div}\left(\mathcal{A}\left(X_{0}, \nabla u_{0}\right)\right)=0 \quad \text { in }\left\{u_{0}>0\right\} .
$$

Since $\partial\left\{u_{0}>0\right\}$ is the smooth surface $\left\{X \in \mathbb{R}^{n}:\left\langle X, \nu\left(X_{0}\right)\right\rangle=0\right\}$ we obtain

$$
\partial\left\{u_{0}>0\right\}=\partial_{\text {red }}\left\{u_{0}>0\right\} .
$$

By Theorem 5.4 we find

$$
\begin{aligned}
-\int_{\Omega^{\prime}}\left\langle\mathcal{A}\left(X_{0}+r_{j} X, \nabla u_{j}\right), \nabla \zeta\right\rangle d X= & r_{j}^{m} \int_{\Omega^{\prime}} m f\left(X_{0}+r_{j} X\right) u_{j}^{m-1} \chi_{\left\{u_{j}>0\right\}} \zeta d X \\
& +\int_{\partial\left\{u_{j}>0\right\} \cap \Omega^{\prime}} \tilde{Q}\left(X_{0}+r_{j} X\right) \zeta d \mathcal{H}^{n-1},
\end{aligned}
$$

for $\zeta \in C_{0}^{\infty}\left(\Omega^{\prime}\right)$, where $\Omega^{\prime} \Subset\left\{\left\langle X, \nu\left(X_{0}\right)\right\rangle<0\right\}$. Now let $\eta \in C_{0}^{\infty}\left(B_{r}^{\prime}\right)$ be a nonnegative function, where $B_{r}^{\prime}$ is a ball in $\mathbb{R}^{n-1}:=\left\{\left\langle X, \nu\left(X_{0}\right)\right\rangle<0\right\}$. By standard arguments (see [1], Theorem 4.8) we have

$$
\int_{\partial\left\{u_{j}>0\right\}} \zeta d \mathcal{H}^{n-1} \rightarrow \int_{\mathbb{R}^{n-1}} \eta d \mathcal{H}^{n-1} .
$$

Furthermore, for some universal constant $C>0$ we have

$$
\left|r_{j}^{m} \int_{\Omega^{\prime}} m f\left(X_{0}+r_{j} X\right) u_{j}^{m-1} \chi_{\left\{u_{j}>0\right\}} \zeta d X\right| \leq r^{m} C,
$$

where we use that the sequence $u_{j}$ is universally bounded. Thus, using (5.25) we obtain

$$
\operatorname{div}\left(\mathcal{A}\left(X_{0}, \nabla u_{0}\right)\right)=\tilde{Q}\left(X_{0}\right) \mathcal{H}^{n-1}\left\lfloor\left\{X \in \mathbb{R}^{n}:\left\langle X, \nu\left(X_{0}\right)\right\rangle=0\right\} .\right.
$$

Moreover, since $\partial_{\text {red }}\left\{u_{0}>0\right\}$ is $C^{1}$ we have

$$
\left\langle\mathcal{A}\left(X_{0}, \nu\left(X_{0}\right)\right), \nu\left(X_{0}\right)\right\rangle=\tilde{Q}\left(X_{0}\right),
$$


for $X_{0}$ at $\partial_{\text {red }}\left\{u_{0}>0\right\}$. However,

$$
\nu\left(X_{0}\right)=\frac{\nabla u\left(X_{0}\right)}{\left|\nabla u\left(X_{0}\right)\right|},
$$

and using (G3) and the identity (5.26) we obtain

$$
\alpha\left(X_{0}\right)=\left(\frac{\tilde{Q}\left(X_{0}\right)}{\left\langle\mathcal{A}\left(X_{0}, \nu\left(X_{0}\right)\right), \nu\left(X_{0}\right)\right\rangle}\right)^{1 /(p-1)}, \quad \forall X \in\left\{\left\langle X, \nu\left(X_{0}\right)\right\rangle=0\right\} .
$$

Hence, we conclude

$$
\nabla u_{0}(X) \cdot \nu\left(X_{0}\right)=\alpha\left(X_{0}\right), \quad \forall X \in\left\{\left\langle X, \nu\left(X_{0}\right)\right\rangle=0\right\} .
$$

Define the function $v_{0}$ by

$$
v_{0}(X)=\left\{\begin{aligned}
u_{0}(X), & \text { if } X \in\left\{\left\langle X, \nu\left(X_{0}\right)\right\rangle<0\right\} \\
-u_{0}\left(X^{*}\right), & \text { if } X \in\left\{\left\langle X, \nu\left(X_{0}\right)\right\rangle \geq 0\right\}
\end{aligned}\right.
$$

where $X^{*}$ is the reflection of $X$ with respect to the hyperplane $\left\{\left\langle X, \nu\left(X_{0}\right)\right\rangle=0\right\}$. Using standard arguments we verify that $v_{0}$ is Lipschitz continuous in $\mathbb{R}^{n}\left(u_{0}\right.$ is Lipschitz continuous by Theorem 4.1) and

$$
\operatorname{div}\left(\mathcal{A}\left(X_{0}, \nabla v_{0}\right)\right)=0 \quad \text { in } \mathbb{R}^{n} .
$$

By the $C^{1, \beta}$ regularity of $v_{0}$, we can apply the blow-up argument from [12] to conclude that $v_{0}$ is an affine function. Then, using (5.27) we find

$$
u_{0}(X)=\alpha\left(X_{0}\right)\left\langle X-X_{0}, \nu\left(X_{0}\right)\right\rangle^{+}
$$

and the result is proved.

Remark 5.8. If

$$
\mathcal{A}(X, \xi)=|\xi|^{p-2} \xi,
$$

that is, in the case of the $p$-Laplacian, we conclude from Lemma 5.6 that the blow-up $u_{0}$ is a minimizer of the functional

$$
\int_{B_{1}(0)}|\nabla v|^{p}+Q\left(X_{0}\right) \chi_{\{v>0\}} d X .
$$

Through a standard Hadamard domain variation argument (see [1], Theorems 2.5 and 5.5 and [6], Theorems 2.1 and 5.6), we obtain the free boundary condition

$$
\alpha\left(X_{0}\right)=\left(\frac{Q\left(X_{0}\right)}{p-1}\right)^{1 / p}
$$

for $X_{0} \in \partial_{\text {red }}\{u>0\}$.

Using (5.23) we have the relation

$$
\tilde{Q}\left(X_{0}\right)=\left(\frac{Q\left(X_{0}\right)}{p-1}\right)^{(p-1) / p} .
$$

between $\tilde{Q}$ and $Q$, for almost every $X_{0} \in \partial_{\text {red }}\{u>0\}$. Taking $p=2$ we find the relation that appears in [1]. 


\section{Jet flow problems and smoothness of the free boundary}

In this section we address the question of the smoothness of the free boundary.

Because our primary motivations come from the theory of heterogeneous jet flows, in this section we shall only treat the nondegenerate problem, i.e., we will work under the assumptions

$$
F(X, \xi)=\frac{1}{2} A(X)|\xi|^{2}+f(X)\left(u^{+}\right)^{m}+Q \chi_{\{u>0\}},
$$

for $X \in \Omega$ and $\xi \in \mathbb{R}^{n}, 1 \leq m<2, f \in C(\Omega)$ and $Q \in C^{0, \beta}, 0<\kappa<Q<\kappa^{-1}$. The matrix $A$ is assumed to be Lipschitz and positive definite.

The proof we will present for smoothness of the reduced free boundary is based on a flatness improvement coming from Harnack type estimates and it follows closely the recent work of [7]. There are a few subtle differences though. For instance the equation we consider is naturally in divergence form, thus it has drift terms in non-divergence form. Also the free boundary condition obtained in (5.23) is a bit more involved than the one treated in [7]. For sake of completeness and the readers' convenience, we shall carry out all the details.

We shall use Caffarelli's viscosity solution setting to address the free boundary regularity theory. We recall some terminologies. Let $u, \phi \in C(\Omega)$. If $u\left(X_{0}\right)=\phi\left(X_{0}\right)$ and there exists a neighborhood $V$ of $X_{0}$ such that

$$
u(X) \geq \phi(X) \quad(\text { respectively } u(X) \leq \phi(X)) \quad \text { in } V,
$$

we say that $\phi$ touches $u$ from below (respectively above) at $X_{0} \in \Omega$. Moreover, if the inequality in (6.2) is strict in $V \backslash\left\{X_{0}\right\}$, we say that $\phi$ touches $u$ strictly from below (resp. above) at $X_{0} \in \Omega$.

The next proposition is standard in Caffarelli's theory ([3], [4], [5]), so we omit its proof.

Proposition 6.1. Assume (6.1). A minimizer $u$ of (1.1) is a viscosity solution of

$$
\begin{cases}\operatorname{div}(A(X) \nabla u)=g(X) & \text { in } \Omega_{+}(u):=\{u>0\}, \\ \langle A \nabla u, \nabla u\rangle=Q & \text { on } \mathcal{F}(u):=\partial\{u>0\} \cap \Omega,\end{cases}
$$

where $g \in L^{\infty}(\Omega) \cap C(\Omega)$. The free boundary condition above is understood in the Caffarelli viscosity sense: if $\phi \in C^{2}(\Omega)$ and $\phi^{+}$touches $u$ from below (respectively from above) at $X_{0} \in \mathcal{F}(u)$ with $|\nabla \phi|\left(X_{0}\right) \neq 0$ then

$$
\left\langle A\left(X_{0}\right) \nabla \phi\left(X_{0}\right), \nabla \phi\left(X_{0}\right)\right\rangle \leq Q\left(X_{0}\right) \quad\left(\text { resp. } \geq Q\left(X_{0}\right)\right) .
$$

The free boundary regularity result we will prove is this section is the following:

Theorem 6.2. Let $u$ be a viscosity solution to (6.3) in the ball $B_{1}(0)$. Suppose that $0 \in \mathcal{F}(u), Q(0)=1$, and $a_{i j}(0)=\delta_{i j}$. There exists a universal constant $\tilde{\varepsilon}>0$ such that, if the graph of $u$ is $\tilde{\varepsilon}$-flat in $B_{1}(0)$, i.e.

$$
\left(X_{n}-\tilde{\varepsilon}\right)^{+} \leq u(X) \leq\left(X_{n}+\tilde{\varepsilon}\right)^{+} \quad \text { for } X \in B_{1}(0),
$$


and

$$
\left[a_{i j}\right]_{C^{0,1}\left(B_{1}(0)\right)} \leq \tilde{\varepsilon}, \quad\|g\|_{L^{\infty}\left(B_{1}(0)\right)} \leq \tilde{\varepsilon}, \quad[Q]_{C^{0, \beta}\left(B_{1}(0)\right)} \leq \tilde{\varepsilon},
$$

then $F(u)$ is $C^{1, \gamma}$ in $B_{1 / 2}(0)$.

Corollary 6.3. Assume (6.1). The reduced free boundary of a minimizer $u$ of (1.1) is locally a $C^{1, \gamma}$ surface. In particular,

$$
\langle A(Z) \nabla u(Z), \nabla u(Z)\rangle=Q(Z)
$$

in the classical sense for $\mathcal{H}^{n-1}$ almost all free boundary points $Z \in \partial\{u>0\}$.

Thus, taking $g(X)=m f(X) u^{m-1}$ we obtain the free boundary regularity theory for our problem. The approach is based fundamentally on comparison criterion.

Definition 6.4. Let $v \in C^{2}(\Omega)$. We say $v$ is a strict (comparison) subsolution (respectively supersolution) to (6.3) in $\Omega$, if the following conditions are satisfied:

1. $\operatorname{div}(A(X) \nabla v)>g(X)$ (resp. $<)$ in $\Omega_{+}(v)$;

2. If $X_{0} \in \mathcal{F}(v)$ then

$$
\langle A \nabla \phi, \nabla \phi\rangle\left(X_{0}\right)>Q\left(X_{0}\right) \quad\left(\text { resp. } 0<\langle A \nabla \phi, \nabla \phi\rangle\left(X_{0}\right)<Q\left(X_{0}\right)\right) .
$$

The next lemma provides a basic comparison principle for solutions to the free boundary problem (6.3).

Lemma 6.5. Let $u$ be a viscosity solution to (6.3) in $\Omega$. If $v$ is a strict subsolution to (6.3) in $\Omega$, such that $u \geq v^{+}$in $\Omega$, then, in $\Omega^{+}(v) \cup \mathcal{F}(v)$, the strict inequality, $u>v^{+}$holds.

Lemma 6.5 yields the crucial tool for the proof of Theorem 6.2. More precisely, using the comparison principle established in Lemma 6.5, we prove a Harnack inequality for solution $u$. For $0<\varepsilon<1$ to be chosen later, we can assume, by normalization and dilating variables, the following conditions:

$$
\begin{aligned}
\left\|a_{i j}-\delta_{i j}\right\|_{L^{\infty}(\Omega)} & \leq \varepsilon^{2}, \\
\|g(X)\|_{L^{\infty}(\Omega)} & \leq \varepsilon^{2}, \\
\left\|\nabla a_{i j}\right\|_{L^{\infty}(\Omega)} & \leq \varepsilon^{2}, \\
\|Q-1\|_{L^{\infty}(\Omega)} & \leq \varepsilon^{2} .
\end{aligned}
$$

We need the following lemma.

Lemma 6.6. Let $u$ be a viscosity solution to (6.3) in $\Omega$, satisfying (6.7)-(6.10). There exists a universal constant $\tilde{\varepsilon}>0$ such that if $0<\varepsilon \leq \tilde{\varepsilon}$ and $u$ satisfies

$$
p^{+}(X) \leq u(X) \leq(p(X)+\sigma)^{+}, \quad|\sigma|<\frac{1}{20} \quad \text { in } \quad B_{1}(0), \quad p(X)=X_{n}+\sigma,
$$

then if at $X_{0}=\frac{1}{10} e_{n}$

$$
u\left(X_{0}\right) \geq\left(p\left(X_{0}\right)+\frac{\varepsilon}{2}\right)^{+}
$$


then

$$
u \geq(p+c \varepsilon)^{+} \text {in } \bar{B}_{1 / 2}(0),
$$

for some $0<c<1$. Analogously, if

$$
u\left(X_{0}\right) \leq\left(p\left(X_{0}\right)+\frac{\varepsilon}{2}\right)^{+},
$$

then

$$
u \leq(p+(1-c) \varepsilon)^{+} \text {in } \bar{B}_{1 / 2}(0) .
$$

Proof. The proof is as in [7]. We will only check the first claim, as the proof of the second is analogous. Let $w: \bar{D} \rightarrow \mathbb{R}$ be defined by

$$
w(X)=c\left(\left|X-X_{0}\right|^{-\gamma}-\left(\frac{4}{5}\right)^{-\gamma}\right),
$$

where $D:=B_{4 / 5}\left(X_{0}\right) \backslash \bar{B}_{1 / 40}\left(X_{0}\right)$. We choose $c>0$ such that

$$
w= \begin{cases}0, & \text { on } \partial B_{4 / 5}\left(X_{0}\right), \\ 1, & \text { on } \partial B_{1 / 40}\left(X_{0}\right) .\end{cases}
$$

We compute directly

$$
\partial_{i} w=-\gamma\left(X_{i}-X_{0}^{i}\right)\left|X-X_{0}\right|^{-\gamma-2}
$$

and

$$
\partial_{i j} w=\gamma\left|X-X_{0}\right|^{-\gamma-2}\left\{(\gamma+2)\left(X_{i}-X_{0}^{i}\right)\left(X_{j}-X_{0}^{j}\right)\left|X-X_{0}\right|^{-2}-\delta_{i j}\right\} .
$$

If we write $b_{i}:=\sum_{j=1}^{n} \partial a_{i j} / \partial x_{i}$, from $\left\|a_{i j}-\delta_{i j}\right\|_{L^{\infty}(\Omega)} \leq \varepsilon^{2}$ and $\left\|b_{i}\right\|_{L^{\infty}(\Omega)} \leq C_{0} \varepsilon^{2}$, we obtain, in $D$, by choosing $\gamma>0$ large,

$$
\begin{gathered}
\operatorname{div}(A(X) \nabla v)=\gamma\left|X-X_{0}\right|^{-\gamma-2}\left\{(\gamma+2)\left|X-X_{0}\right|^{-2} \sum_{i, j=1}^{n} a_{i j}(X)\left(X_{i}-X_{0}^{i}\right)\right. \\
\left.\cdot\left(X_{j}-X_{0}^{j}\right)-\sum_{i, j=1}^{n} a_{i j}(X) \delta_{i j}-\sum_{i=1}^{n} b_{i}(X)\left(X_{i}-X_{0}^{i}\right)\right\} \\
(6.17) \quad \geq \gamma\left|X-X_{0}\right|^{-\gamma-2}\{(\gamma+2)-C(n)\} \geq \delta_{0},
\end{gathered}
$$

where $\delta_{0}>0$ is a universal constant. From (6.11) we have $u \geq p$ in $B_{1}(0)$. Thus,

$$
B_{1 / 20}\left(X_{0}\right) \subset B_{1}^{+}(u) .
$$

Moreover,

$$
\operatorname{div}(A(X) \nabla(u-p))=\operatorname{div}(A(X) \nabla u)-b_{n}=g(X)-b_{n}, \quad \text { in } B_{1 / 20}\left(X_{0}\right),
$$

with

$$
\left\|g(X)-b_{n}\right\|_{L^{\infty}\left(B_{1 / 20}\left(X_{0}\right)\right)} \leq C \varepsilon^{2} .
$$


Hence, by the Harnack inequality, we obtain

$$
\begin{aligned}
u(X)-p(X) & \geq u\left(X_{0}\right)-p\left(X_{0}\right)-C\left\|g(X)-b_{n}\right\|_{L^{\infty}\left(B_{1 / 20}\left(X_{0}\right)\right)} \\
& \geq u\left(X_{0}\right)-p\left(X_{0}\right)-C \varepsilon^{2},
\end{aligned}
$$

for all $X \in B_{1 / 40}\left(X_{0}\right)$. Using (6.11) for $\varepsilon$ sufficiently small,

$$
u(X)-p(X) \geq c \varepsilon-C \varepsilon^{2} \geq c_{0} \varepsilon, \quad \text { in } B_{1 / 40}\left(X_{0}\right) .
$$

Define

$$
v(X)=p(X)+c_{0} \varepsilon(w(X)-1), \quad X \in \bar{B}_{4 / 5}\left(X_{0}\right),
$$

and for $t \geq 0$,

$$
v_{t}(X)=v(X)+t, \quad X \in \bar{B}_{4 / 5}\left(X_{0}\right) .
$$

By the maximum principle (see (6.16) and (6.17)) we have $w \leq 1$ in $D$. Then, extending $w$ to 1 in $B_{1 / 40}\left(X_{0}\right)$ we find

$$
v_{0}(X)=v(X) \leq p(X) \leq u(X), \quad X \in \bar{B}_{4 / 5}\left(X_{0}\right) .
$$

Consider

$$
t_{0}=\sup \left\{t \geq 0: v_{t} \leq u \quad \text { in } \bar{B}_{4 / 5}\left(X_{0}\right)\right\} .
$$

Assume, for the moment, that we have already verified $t_{0} \geq c_{0} \varepsilon$. From the definition of $v$ we have

$$
u(X) \geq v(X)+t_{0} \geq p(X)+c_{0} \varepsilon w(X), \quad \forall X \in B_{4 / 5}\left(X_{0}\right) .
$$

Notice that $B_{1 / 2}(0) \subset B_{3 / 5}\left(X_{0}\right)$ and

$$
w(X) \geq \begin{cases}\left(\frac{3}{5}\right)^{-\gamma}-\left(\frac{4}{5}\right)^{-\gamma}, & \text { in } B_{3 / 5}\left(X_{0}\right) \backslash B_{1 / 40}\left(X_{0}\right), \\ 1, & \text { on } B_{1 / 40}\left(X_{0}\right) .\end{cases}
$$

Hence, we conclude ( $\varepsilon$ small) that

$$
u(X)-p(X) \geq c \varepsilon, \quad \text { in } B_{1 / 2}(0),
$$

and the result is proved.

Now we prove that indeed $t_{0} \geq c_{0} \varepsilon$. For this, we suppose for the sake of contradiction, that $t_{0}<c_{0} \varepsilon$. Then there would exist $Y_{0} \in \bar{B}_{4 / 5}\left(X_{0}\right)$ such that

$$
v_{t}\left(Y_{0}\right)=u\left(Y_{0}\right)
$$

In the sequel, we show that $Y_{0} \in B_{1 / 40}\left(X_{0}\right)$. From definition of $v_{t}$ and by the fact that $w$ has zero boundary values on $\partial B_{4 / 5}\left(X_{0}\right)$ we have

$$
v_{t}=p-c_{0} \varepsilon+t_{0}<u \quad \text { in } \partial B_{4 / 5}\left(X_{0}\right),
$$

where we have used that $u \geq p$ and $t_{0}<c_{0} \varepsilon$. Moreover,

$$
\operatorname{div}\left(A(X) \nabla v_{t}\right) \geq\left(c_{0} \delta_{0}-\varepsilon\right) \varepsilon>\varepsilon^{2} \quad \text { in } D
$$


and

$$
\left|\nabla v_{t_{0}}\right| \geq\left|\partial_{n} v\right|=\left|1+c_{0} \varepsilon \partial_{n} w\right|, \quad \text { in } D .
$$

By the radial symmetry of $w$, we have

$$
\partial_{n} w(X)=|\nabla w(X)|\left\langle\nu_{X}, e_{n}\right\rangle, \quad X \in D,
$$

where $\nu_{X}$ is the unit vector in the direction of $X-X_{0}$. From (6.17) we have

$$
|\nabla w|^{2}=\gamma^{2}\left|X-X_{0}\right|^{-2(\gamma+2)}\left|X-X_{0}\right|^{2}=\gamma^{2}\left|X-X_{0}\right|^{-2(\gamma+1)} \geq c>0, \quad \text { in } D \text {. }
$$

Also we have $\left\langle\nu_{X}, e_{n}\right\rangle \geq c$ in $\left\{v_{t_{0}} \leq 0\right\} \cap D$ (for $\varepsilon$ small enough). In fact, if $\varepsilon$ is small enough

$$
\left\{v_{t_{0}} \leq 0\right\} \cap D \subset\left\{p \leq c_{0} \varepsilon\right\}=\left\{X_{n} \leq c_{0} \varepsilon-\sigma\right\} \subset\left\{X_{n}<1 / 20\right\} .
$$

We therefore conclude that

$$
\begin{aligned}
\left\langle\nu_{X}, e_{n}\right\rangle & =\frac{1}{\left|X_{0}-X\right|}\left\langle X-X_{0}, e_{n}\right\rangle \geq \frac{5}{4}\left\langle X-X_{0}, e_{n}\right\rangle \\
& =\frac{5}{4}\left(-X_{n}+\frac{1}{20}-\frac{1}{20}+\frac{1}{10}\right)>\frac{1}{16}, \quad \text { in } \quad\left\{v_{t_{0}} \leq 0\right\} \cap D .
\end{aligned}
$$

Moreover, from $\left\|a_{i j}-\delta_{i j}\right\| \leq \varepsilon^{2}$ we have

$$
\langle A(X) \xi, \xi\rangle \geq|\xi|^{2}\left(1-\varepsilon^{2}\right), \quad \forall X \in \Omega, \forall \xi \in \mathbb{R}^{n} .
$$

Therefore, from (6.23), (6.24) and (6.25) we obtain

$$
\left\langle A \nabla v_{t_{0}}, \nabla v_{t_{0}}\right\rangle \geq\left|\nabla v_{t_{0}}\right|^{2}-C \varepsilon^{2} \geq 1+c_{1} \varepsilon+\varepsilon\left(c_{1}-C \varepsilon\right)+c_{1}^{2} \varepsilon^{2}>1+\varepsilon^{2}>Q,
$$

in $\left\{v_{t_{0}} \leq 0\right\} \cap D$. In particular, we have

$$
\left\langle A \nabla v_{t_{0}}, \nabla v_{t_{0}}\right\rangle>Q \quad \text { in } D \cap \mathcal{F}\left(v_{t_{0}}\right) .
$$

Thus, $v_{t_{0}}$ is a strict subsolution in $D$ and by Lemma 6.5 ( $u$ is a viscosity solution of problem (6.3) in $\left.B_{1}(0)\right)$ we conclude that $Y_{0} \in B_{1 / 40}\left(X_{0}\right)$. This is a contradiction. In fact, we would get

$$
u\left(Y_{0}\right)=v_{t_{0}}\left(Y_{0}\right)=v\left(Y_{0}\right)+t_{0} \leq p\left(Y_{0}\right)+t_{0}<p\left(Y_{0}\right)+c_{0} \varepsilon .
$$

which leads to a contradiction with (6.19). This concludes the proof of the lemma.

We can now establish the main tool needed for the proof of Theorem 6.2.

Theorem 6.7. Let $u$ be a viscosity solution to (6.3) in $\Omega$ satisfying (6.7)-(6.10). There exists a universal constant $\tilde{\varepsilon}>0$ such that if $u$ satisfies

$$
\left(X_{n}+a_{0}\right)^{+} \leq u(X) \leq\left(X_{n}+d_{0}\right)^{+} \quad \text { in } B_{r}\left(X_{0}\right) \subset \Omega,
$$

at some $X_{0} \in \Omega^{+}(u) \cup F(u)$, with

$$
d_{0}-a_{0} \leq \varepsilon r, \quad \varepsilon \leq \tilde{\varepsilon},
$$


then

$$
\left(X_{n}+a_{1}\right)^{+} \leq u(X) \leq\left(X_{n}+d_{1}\right)^{+} \quad \text { in } B_{r / 40}\left(X_{0}\right)
$$

with

$$
a_{0} \leq a_{1} \leq d_{1} \leq d_{0}, \quad d_{1}-a_{1} \leq(1-c) \varepsilon r,
$$

and $0<c<1$ universal.

Proof. With no loss of generality, we assume $X_{0}=0$ and $r=1$. We analyze two distinct cases.

1) $\left|a_{0}\right|<1 / 20$ : We put $p(X)=X_{n}+a_{0}$ and by $(6.26)$

$$
p^{+}(X) \leq u(X) \leq\left(p(X)+a_{0}\right)^{+}\left(d_{0} \leq a_{0}+\varepsilon\right) .
$$

Thus, we can apply Lemma 6.6 to obtain the result.

2) $\left|a_{0}\right| \geq 1 / 20$ : Assume that $a_{0}<-1 / 20$. If we take $\varepsilon<1 / 20$, it follows that $(p+\varepsilon)^{+}=0$. Thus 0 belongs to the interior of the zero phase of $u$, which yields a contradiction.

If $a_{0}>1 / 20$, then $B_{1 / 20}(0) \subset B_{1}^{+}(u)$ and the result follows from the Harnack inequality. In fact, if $p=x_{n}+d_{0}$

$$
\operatorname{div}(A(X) \nabla(p-u))=b_{n}-g(X),
$$

with $\left\|b_{n}-g(X)\right\|_{L^{\infty}\left(B_{1 / 20}(0)\right)} \leq C \varepsilon^{2}$. Hence, since $(p-u)(0)=a_{0}$ we have

$$
p(X)-u(X) \geq c_{0} d_{0}-C \varepsilon^{2}, \quad\left(0<c_{1}<1\right)
$$

which implies

$$
u(X) \leq p(X)-d_{0} c_{0}+C \varepsilon^{2}=X_{n}+d_{0}\left(1-c_{0}\right)+C \varepsilon^{2}, \quad \forall X \in B_{1 / 40}\left(X_{0}\right) .
$$

Let $c_{1}=d_{0}-a_{0}$. Then,

$$
\begin{aligned}
u(X) & \leq X_{n}+\left(c_{1}+a_{0}\right)\left(1-c_{0}\right)+C \varepsilon^{2}=X_{n}+a_{0}+c_{1}\left(1-c_{0}\right)-a_{0} c_{0}+C \varepsilon^{2} \\
& \leq X_{n}+a_{0}+c_{1}\left(1-c_{0}\right)-\frac{c_{0}}{20}+C \varepsilon^{2} \leq X_{n}+a_{0}+c_{1}\left(1-c_{0}\right),
\end{aligned}
$$

if $\varepsilon$ is small enough. Hence, if we put $a_{1}:=a_{0}$ and $d_{1}=a_{0}+c_{1}\left(1-c_{0}\right)$ we obtain the result.

From the Harnack inequality, Theorem 6.7, we obtain precisely as in [7] the following key estimate for flatness improvement.

Corollary 6.8. Let $u$ be a viscosity solution to (6.3) in $\Omega$ satisfying (6.7)-(6.10). If $u$ satisfies (6.26) then in $B_{1}\left(X_{0}\right)$ the function $\tilde{u}_{\varepsilon}:=\left(u-X_{n}\right) / \varepsilon$ has a Hölder modulus of continuity at $X_{0}$ outside of a ball of radius $\varepsilon / \tilde{\varepsilon}$, i.e., for all $X \in$ $\left(\Omega^{+}(u) \cup F(u)\right) \cap B_{1}\left(X_{0}\right)$ with $\left|X-X_{0}\right| \geq \varepsilon / \tilde{\varepsilon}$,

$$
\left|\tilde{u}_{\varepsilon}(X)-\tilde{u}_{\varepsilon}\left(X_{0}\right)\right| \leq C\left|X-X_{0}\right|^{\gamma} .
$$


We are ready to establish improvement of flatness.

Theorem 6.9 (Flatness improvement). Let $u$ be a viscosity solution to (6.3) in $\Omega$ satisfying (6.7)-(6.10). Assume that u satisfies

$$
\left(X_{n}-\varepsilon\right)^{+} \leq u \leq\left(X_{n}+\varepsilon\right)^{+} \quad \text { for } X \in B_{1}(0),
$$

with $0 \in \mathcal{F}(u)$. If $0<r \leq r_{0}$ for a universal constant $r_{0}$ and $0<\varepsilon \leq \varepsilon_{0}(r)$, then

$$
\left(X \cdot \nu-\frac{r}{2} \varepsilon\right)^{+} \leq u \leq\left(X \cdot \nu+\frac{r}{2} \varepsilon\right)^{+} \quad \text { for } X \in B_{r}(0),
$$

with $|\nu|=1$, and $\left|\nu-e_{n}\right| \leq C \varepsilon^{2}$ for a universal constant $C$.

Proof. Again the proof follows the lines of [7]. Fix $0<r \leq r_{0}$ with $r_{0}$ a constant to be chosen. Aiming at contradiction, suppose that there exist a sequence $\varepsilon_{j} \rightarrow 0$ and a sequence $u_{j}$ of solutions to (6.3) in $B_{1}(0)$ with right-hand side $g_{j}$ and free boundary condition $Q_{j}$ such that

$$
\left(X_{n}-\varepsilon_{j}\right)^{+} \leq u_{j} \leq\left(X_{n}-\varepsilon_{j}\right)^{+} \text {for } X \in B_{1}(0), 0 \in \mathcal{F}\left(u_{j}\right),
$$

but $u_{j}$ does not satisfy the conclusion (6.32). Define

$$
\tilde{u}_{j}(X)=\frac{u_{j}(X)-X_{n}}{\varepsilon_{j}}, \quad X \in \Omega_{1}\left(u_{j}\right),
$$

where $\Omega_{\rho}:=\left(B_{1}^{+}(u) \cup F(u)\right) \cap B_{\rho}(0)$, for $0<\rho<1$. Then (by Corollary 5.8) the graphs of the $\tilde{u}_{j}$ over $\Omega_{\frac{1}{2}}\left(u_{j}\right)$ converge (possibly passing to a subsequence) in the Hausdorff distance to the graph of a Hölder continuous function $\tilde{u}$ over $B_{1 / 2}(0) \cap\left\{X_{n} \geq 0\right\}$. We claim that $\tilde{u}$ is a solution of the problem

$$
\begin{cases}\Delta \tilde{u}=0 & \text { in } B_{1 / 2}(0) \cap\left\{X_{n}>0\right\}, \\ \partial_{n} \tilde{u}=0 & \text { on } B_{1 / 2}(0) \cap\left\{X_{n}=0\right\},\end{cases}
$$

in the viscosity sense (see Definition 2.5 and the subsequent remark in [7]). Given a quadratic polynomial $P(X)$ touching $\tilde{u}$ at $X_{0} \in B_{1 / 2}(0) \cap\left\{X_{n} \geq 0\right\}$ strictly from below we need to prove that

(i) if $X_{0} \in B_{1 / 2}(0) \cap\left\{X_{n}>0\right\}$ then $\Delta P \leq 0$;

(ii) if $X_{0} \in B_{1 / 2}(0) \cap\left\{X_{n}=0\right\}$ then $\partial_{n} P\left(X_{0}\right) \leq 0$.

As in [7], there exist points $X_{j} \in \Omega_{1 / 2}\left(u_{j}\right), X_{j} \rightarrow X_{0}$, and constants $c_{j} \rightarrow 0$ such that

$$
u_{j}\left(X_{j}\right)=\tilde{P}\left(X_{j}\right) \quad \text { and } \quad u_{j}(X) \geq \tilde{P}(X) \quad \text { in a neighborhood of } X_{j}
$$

where

$$
\tilde{P}(X)=\varepsilon_{j}\left(P(X)+c_{j}\right)+X_{n} .
$$

We have two possibilities: 
(a) If $X_{0} \in B_{1 / 2}(0) \cap\left\{X_{n}>0\right\}$ then, since $P$ touches $u_{j}$ from below at $X_{j}$, we obtain

$$
\begin{aligned}
C_{1} \varepsilon_{j}^{2} \geq g_{j}\left(X_{j}\right) & \geq \sum_{i, l=1}^{n} a_{i l}^{j}(X) \partial_{i l} \tilde{P}+\sum_{i=1}^{n} b_{i}^{j}\left(X_{j}\right) \partial_{i} \tilde{P} \\
& =\varepsilon_{j} \sum_{i, l=1}^{n} a_{i l}^{j}\left(X_{j}\right) \partial_{i l} P+\varepsilon_{j} \sum_{i=1}^{n} b_{i}^{j}\left(X_{j}\right) \partial_{i} P+b_{n}^{j}\left(X_{j}\right),
\end{aligned}
$$

where $\left\|b_{i}^{j}\right\|_{L^{\infty}} \leq C_{0} \varepsilon_{j}^{2}$ and $\left\|\partial_{i} P\right\|_{L^{\infty}} \leq C$. Therefore,

$$
\sum_{i, l=1}^{n} a_{i l}^{j}\left(X_{j}\right) \partial_{i l} P \leq C \varepsilon_{j}
$$

Thus, we have

$$
\Delta P=\sum_{i, l=1}^{n}\left(\delta_{i l}-a_{i l}^{j}\left(X_{k}\right)\right) \partial_{i l} P+\sum_{i, l=1}^{n} a_{i l}^{j}\left(X_{k}\right) \partial_{i l} P \leq C \varepsilon_{j} .
$$

Hence, taking $j \rightarrow \infty$ we obtain $\Delta P \leq 0$.

(b) If $X_{0} \in B_{1 / 2}(0) \cap\left\{X_{n}=0\right\}$ we can assume (see [7]) that

$$
\Delta P>0
$$

Notice that for $j$ sufficiently large we have $X_{j} \in F\left(u_{j}\right)$. In fact, aiming at a contradiction, suppose that there exists a subsequence $X_{j_{n}} \in B_{1}^{+}\left(u_{j_{n}}\right)$ such that $X_{j_{n}} \rightarrow X_{0}$. Then, arguing as in (a), we obtain

$$
\Delta P \leq C \varepsilon_{j},
$$

which contradicts (6.34) as $j_{n} \rightarrow \infty$. Therefore, there exists $j_{0} \in \mathbb{N}$ such that $X_{j} \in F\left(u_{j}\right)$ for $j \geq j_{0}$. Moreover,

$$
|\nabla \tilde{P}| \geq 1-\varepsilon_{j}|\nabla P|>0,
$$

for $j$ sufficiently large (we can assume that $j \geq j_{0}$ ). Since that $\tilde{P}^{+}$touches $u_{j}$ from below we have

$$
\left\langle A^{j}\left(X_{j}\right) \nabla \tilde{P}\left(X_{j}\right), \nabla \tilde{P}\left(X_{j}\right)\right\rangle \leq Q_{j}\left(X_{j}\right) \leq\left(1+\varepsilon_{j}^{2}\right) .
$$

Moreover,

$$
\begin{aligned}
\left\langle A^{j}\left(X_{j}\right) \nabla \tilde{P}\left(X_{j}\right), \nabla \tilde{P}\left(X_{j}\right)\right\rangle & \geq\left|\nabla \tilde{P}\left(X_{j}\right)\right|^{2}-C \varepsilon_{j}^{2} \\
& =\varepsilon_{j}^{2}\left|\nabla P\left(X_{j}\right)\right|^{2}+1+2 \varepsilon_{j} \partial_{n} P\left(X_{j}\right)-C \varepsilon_{j}^{2},
\end{aligned}
$$

where we have used $|\nabla \tilde{P}|^{2} \leq C$. In conclusion, we obtain

$$
\varepsilon_{j}^{2}\left|\nabla P\left(X_{j}\right)\right|^{2}+1+2 \varepsilon_{j} \partial_{n} P\left(X_{j}\right)-C \varepsilon_{j}^{2} \leq 1+\varepsilon_{j}^{2} .
$$

Hence, dividing (6.35) by $\varepsilon_{j}$ and taking $j \rightarrow \infty$ we obtain $\partial_{n} P\left(X_{0}\right) \leq 0$.

The choice of $r_{0}$ and the conclusion of the theorem follows from the regularity of $\tilde{u}$. 
We can finally conclude the proof of Theorem 6.2.

Proof of Theorem 6.2. Let

$$
u_{j}(X)=\frac{u\left(\rho_{j} X\right)}{\rho_{j}}, \quad X \in B_{1}(0),
$$

be the rescaling of $u$ with $\rho_{j}=\tilde{r}^{j}$ for a fixed $\tilde{r}$ such that

$$
\tilde{r}^{\beta} \leq \frac{1}{4} \quad \text { and } \quad \tilde{r} \leq r_{0}
$$

where $r_{0}$ is the universal constant as in Theorem 6.9. Note that $u_{j}$ is solution of (6.3) with

$$
\begin{aligned}
a_{i l}^{j}(X) & :=a_{i l}^{j}\left(\rho_{j} X\right), \\
g_{j}(X) & :=g\left(\rho_{j} X\right), \\
Q_{j}(X) & :=Q\left(\rho_{j} X\right) .
\end{aligned}
$$

Moreover, if $\tilde{\varepsilon}:=\varepsilon_{0}^{2}(\tilde{r})$ and $\varepsilon_{j}:=2^{-j} \varepsilon_{0}(\tilde{r})$ we obtain

$$
\begin{aligned}
\left|a_{i l}^{j}(X)-\delta_{i l}\right| & =\left|a_{i l}\left(\rho_{j} X\right)-a_{i l}(0)\right| \leq\left[a_{i l}\right]_{C^{0,1}} \rho_{j} \leq \tilde{\varepsilon} \tilde{r}^{j} \leq \varepsilon_{j}^{2}, \\
\left\|b_{i}^{j}\right\|_{L^{\infty}} & \leq\left[a_{i j}\right]_{C^{0,1}} \rho_{j} \leq C_{0} \tilde{\varepsilon} \tilde{r}^{j} \leq \varepsilon_{j}^{2}, \\
\left\|g_{j}\right\|_{L^{\infty}} & \leq\left\|g_{j}\right\|_{L^{\infty}} \rho_{j} \leq \tilde{\varepsilon} \tilde{r}^{j} \leq \varepsilon_{j}^{2} \\
\left|Q_{j}(X)-1\right| & =\left|Q\left(\rho_{j} X\right)-Q(0)\right| \leq[Q]_{C^{0, \beta}} \rho_{j}^{\beta} \leq \tilde{\varepsilon} \tilde{r}^{j \beta} \leq \varepsilon_{j}^{2} .
\end{aligned}
$$

The proof now follows as in [7].

\section{References}

[1] Alt, H. W. And Caffarelli, L. A.: Existence and regularity for a minimum problem with free boundary. J. Reine Angew. Math. 325 (1981), 105-144.

[2] Boccardo, L. ANd Murat, F.: Almost everywhere convergence of the gradients of solutions to elliptic and parabolic equations. Nonlinear Anal. 19 (1992), no. $6,581-597$.

[3] Caffarelli, L. A.: A Harnack inequality approach to the regularity of free boundaries. I. Lipschitz free boundaries are $C^{1, \alpha}$. Rev. Mat. Iberoamericana 3 (1987), no. $2,139-162$.

[4] Caffarelli, L. A.: A Harnack inequality approach to the regularity of free boundaries. II. Flat free boundaries are Lipschitz. Comm. Pure Appl. Math. 42 (1989), no. $1,55-78$.

[5] Caffarelli, L. A.: A Harnack inequality approach to the regularity of free boundaries. III. Existence theory, compactness, and dependence on X. Ann. Scuola Norm. Sup. Pisa Cl. Sci. (4) 15 (1988), no. 4, 583-602 (1989). 
[6] Danielli, D. And Petrosyan, A.: A minimum problem with free boundary for a degenerate quasilinear operator. Calc. Var. Partial Differential Equations 23 (2005), no. $1,97-124$.

[7] De Silva, D.: Free boundary regularity for a problem with right hand side. Interfaces Free Bound. 13 (2011), no. 2, 223-238.

[8] DiBenedetto, E.: $C^{1+\alpha}$ local regularity of weak solutions of degenerate elliptic equations. Nonlinear Anal. 7 (1983), no. 8, 827-850.

[9] Federer, H.: Geometric measure theory. Die Grundlehren der mathematischen Wissenschaften 153, Springer-Verlag New York, New York, 1969.

[10] Gilbarg, D. And Trudinger, N. S.: Elliptic partial differential equations of second order. Second edition. Fundamental Principles of Mathematical Sciences 224, Springer-Verlag, Berlin, 1983.

[11] Heinonen, J., Kilpeläinen, T. and Martio, O.: Nonlinear potential theory of degenerate elliptic equations. Dover Publications, Mineola, NY, 2006.

[12] Kilpeläinen, T., Shahgholian, H. And Zhong, X.: Growth estimates through scaling for quasilinear partial differential equations. Ann. Acad. Sci. Fenn. Math. 32 (2007), no. 2, 595-599.

[13] Ladyzhenskaya, O. A. and Ural'tseva, N. N.: Linear and quasilinear elliptic equations. Academic Press, New York-London, 1968.

[14] Peral, I.: Multiplicity of solutions for the p-Laplacian. Second School of Nonlinear Functional Analysis and Applications to Differential Equations, 1997.

[15] Serrin, J.: A Harnack inequality for nonlinear equations. Bull. Amer. Math. Soc. 69 (1963), 481-486.

[16] TeixeirA, E. V.: Optimal design problems in rough inhomogeneous media: existence theory. Amer. J. Math. 132 (2010), no. 6, 1445-1492.

[17] TeixeirA, E. V.: Sharp regularity for general Poisson equations with borderline sources. J. Math. Pures Appl. (9) 99 (2013), no. 2, 150-164.

[18] TeixeirA, E. V.: Regularity for quasilinear equations on degenerate singular sets. Math. Ann. 358 (2014), no. 1-2, 241-256.

[19] Tolksdorf, P.: Regularity for a more general class of quasilinear elliptic equations. J. Differential Equations 51 (1984), no. 1, 126-150.

Received January 25, 2013.

Eduardo V. Teixeira: Universidade Federal do Ceará, Campus do Pici, Bloco 914, 60.455-760 Fortaleza-CE, Brazil.

E-mail: teixeira@mat.ufc.br

Raimundo Leitão: Universidade Federal do Ceará, Campus do Pici, Bloco 914, 60.455-760 Fortaleza-CE, Brazil.

E-mail: rleitao@mat.ufc.br

This work was partially supported by Capes and CNPq. 